\title{
Rabi oscillations and Ramsey-type pulses in ultracold bosons: Role of interactions
}

\author{
Q. Guan, ${ }^{1,2}$ T. M. Bersano, ${ }^{3}$ S. Mossman, ${ }^{3}$ P. Engels,${ }^{3}$ and D. Blume ${ }^{1,2}$ \\ ${ }^{1}$ Homer L. Dodge Department of Physics and Astronomy, \\ The University of Oklahoma, 440 W. Brooks Street, Norman, Oklahoma 73019, USA \\ ${ }^{2}$ Center for Quantum Research and Technology, The University of Oklahoma, \\ 440 W. Brooks Street, Norman, Oklahoma 73019, USA \\ ${ }^{3}$ Department of Physics and Astronomy, Washington State University, Pullman, Washington 99164-2814, USA
}

(Dated: June 25, 2020)

\begin{abstract}
Double-well systems loaded with one, two, or many quantum particles give rise to intriguing dynamics, ranging from Josephson oscillation to self-trapping. This work presents theoretical and experimental results for two distinct double-well systems, both created using dilute rubidium BoseEinstein condensates with particular emphasis placed on the role of interaction in the systems. The first is realized by creating an effective two-level system through Raman coupling of hyperfine states. The second is an effective two-level system in momentum space generated through the coupling by an optical lattice. Even though the non-interacting systems can, for a wide parameter range, be described by the same model Hamiltonian, the dynamics for these two realizations differ in the presence of interactions. The difference is attributed to scattering diagrams that contribute in the lattice coupled system but vanish in the Raman coupled system. The internal dynamics of the Bose-Einstein condensates for both coupling scenarios is probed through a Ramsey-type interference pulse sequence, which constitutes a key building block of atom interferometers. These results have important implications in a variety of contexts including lattice calibration experiments and momentum space lattices used for quantum analog simulations.
\end{abstract}

PACS numbers:

\section{INTRODUCTION}

We consider the famous model in which two isolated states are resonantly coupled by a monochromatic field, e.g., two energetically separated atomic states that are coupled by an oscillating electric field. Starting with all particles populating one of the states, the population oscillates periodically between the two states under the influence of the external field. In the presence of a positive or negative detuning $\delta$, the population still oscillates back and forth; however, the oscillation period and the maximum transfer probability (amplitude) of these Rabi oscillations are modified. This coupled two-level system, which finds applications in many areas of physics, is discussed in nearly every quantum text book $1+3$. For an interacting ensemble of particles, the Rabi oscillations are, in general, further modified. In particular, the population oscillations may not be fully periodic and the amplitude of the oscillations may decrease or drift (dephase) due to many-body effects [4 6 ].

This work considers Rabi oscillations in the context of ultracold atoms, specifically a degenerate ${ }^{87} \mathrm{Rb}$ BoseEinstein condensate (BEC). The two-level system is realized in two different ways. In scenario 1, one-dimensional Raman coupling along the $z$-direction, realized using two Raman lasers, generates an effective pseudo-spin- $1 / 2$ system of two coupled internal hyperfine states $7-9$. In scenario 2 , a one-dimensional moving optical lattice along the $z$-direction couples momentum states with momenta of $2 n \hbar k_{L}$, where $n$ is an integer and $k_{L}$ the lattice wave vector [10 12]. Considering only the $n=0$ and $n=1$ states, the lattice Rabi coupling case can, in the absence of interactions, be mapped to the same two-state description as the Raman coupling case considered in scenario 1, with the velocity of the moving lattice determining the effective detuning.

In the presence of atom-atom interactions, the Rabi oscillations for scenarios 1 and 2 are found to differ. The reason for this is traced back to how the two-level systems are realized. In second quantization, the interaction potential takes the form

$$
\hat{v}=\int \hat{\Psi}^{\dagger}\left(\mathbf{r}_{1}\right) \hat{\Psi}^{\dagger}\left(\mathbf{r}_{2}\right) V\left(\mathbf{r}_{1}, \mathbf{r}_{2}\right) \hat{\Psi}\left(\mathbf{r}_{1}\right) \hat{\Psi}\left(\mathbf{r}_{2}\right) d \mathbf{r}_{1} d \mathbf{r}_{2},
$$

where $\hat{\Psi}^{\dagger}(\mathbf{r})$ creates a particle at position $\mathbf{r}$. Parametrizing $V\left(\mathbf{r}_{1}, \mathbf{r}_{2}\right)$ in terms of a contact interaction of strength $g, V\left(\mathbf{r}_{1}, \mathbf{r}_{2}\right)=g \delta\left(\mathbf{r}_{1}-\mathbf{r}_{2}\right)$, and assuming that the field operator $\hat{\Psi}^{\dagger}(\mathbf{r})$ can be expressed in terms of two states,

$$
\hat{\Psi}^{\dagger}(\mathbf{r})=\hat{c}_{a}^{\dagger} \Psi_{a}^{*}(\mathbf{r})+\hat{c}_{b}^{\dagger} \Psi_{b}^{*}(\mathbf{r})
$$

$\left(\hat{c}_{a}^{\dagger}\right.$ and $\hat{c}_{b}^{\dagger}$ create a particle in states $\Psi_{a}$ and $\Psi_{b}$, respectively), it can be seen that $\hat{v}$ contains 16 terms. Some of these vanish in the Raman coupling case but contribute appreciably in the lattice coupling case.

We write $\Psi_{a}(\mathbf{r})=\psi_{a}(\mathbf{r})|\uparrow\rangle$ and $\Psi_{b}(\mathbf{r})=\psi_{b}(\mathbf{r})|\downarrow\rangle$. In the Raman coupling case, $|\uparrow\rangle$ and $|\downarrow\rangle$ represent two different hyperfine states and $\psi_{a}(\mathbf{r})$ and $\psi_{b}(\mathbf{r})$ correspond to states with momentum 0 and $2 \hbar k_{R}\left(\hbar k_{R}\right.$ is the Raman momentum). The resulting non-vanishing interaction terms or scattering diagrams are shown in the first row of Fig. 1. The first scattering diagram is proportional to $\hat{c}_{a}^{\dagger} \hat{c}_{a}^{\dagger} \hat{c}_{a} \hat{c}_{a}$, the second scattering diagram is proportional to $\hat{c}_{b}^{\dagger} \hat{c}_{b}^{\dagger} \hat{c}_{b} \hat{c}_{b}$, and the third and fourth scattering 

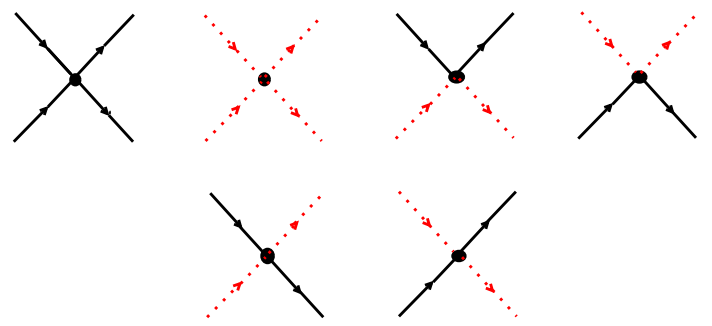

FIG. 1: Scattering diagrams. The scattering diagrams in the first row contribute in the Raman and lattice coupling cases. The scattering diagrams in the second row vanish in the Raman coupling case. In the first diagram of the first row, particle 1 (top incoming arrow) and particle 2 (bottom incoming arrow) are both in the same state (both arrows are black solid); the scattering process does not change the state (the arrows are black solid after the scattering has occured). In the first diagram of the second row, particle 1 (top incoming arrow) and particle 2 (bottom incoming arrow) are in different states (black solid and red dotted arrows, respectively); after the scattering process, the states of particle 1 (top outgoing arrow) and particle 2 (bottom outgoing arrow) are changed (red dotted and black solid arrows, respectively).

diagrams are proportional to $\hat{c}_{a}^{\dagger} \hat{c}_{b}^{\dagger} \hat{c}_{a} \hat{c}_{b}$. The last two processes can be written as $\left|\Psi_{a}\right\rangle_{1}+\left|\Psi_{b}\right\rangle_{2} \rightarrow\left|\Psi_{a}\right\rangle_{1}+\left|\Psi_{b}\right\rangle_{2}$ and $\left|\Psi_{b}\right\rangle_{1}+\left|\Psi_{a}\right\rangle_{2} \rightarrow\left|\Psi_{b}\right\rangle_{1}+\left|\Psi_{a}\right\rangle_{2}$, where the notation $\left|\Psi_{a}\right\rangle_{1}$ means that particle 1 occupies state $\Psi_{a}$. The "mixed scattering diagrams" that are shown in the second row of Fig. 1. which are also proportional to $\hat{c}_{a}^{\dagger} \hat{c}_{b}^{\dagger} \hat{c}_{a} \hat{c}_{b}$, vanish in the Raman coupling case due to the orthogonality of the two hyperfine states $|\uparrow\rangle$ and $|\downarrow\rangle$. They correspond to the processes $\left|\Psi_{a}\right\rangle_{1}+\left|\Psi_{b}\right\rangle_{2} \rightarrow\left|\Psi_{b}\right\rangle_{1}+\left|\Psi_{a}\right\rangle_{2}$ and $\left|\Psi_{b}\right\rangle_{1}+\left|\Psi_{a}\right\rangle_{2} \rightarrow\left|\Psi_{a}\right\rangle_{1}+\left|\Psi_{b}\right\rangle_{2}$.

In the lattice system, all the atoms occupy the same hyperfine state and $\Psi_{a}(\mathbf{r})$ and $\Psi_{b}(\mathbf{r})$ correspond to states with momentum $\approx 0$ and $\approx 2 \hbar k_{L}$, respectively. In this case, $|\uparrow\rangle$ and $|\downarrow\rangle$ represent two different plane wave states and $\Psi_{a}(\mathbf{r})$ and $\Psi_{b}(\mathbf{r})$ are not orthogonal to each other. As a consequence, the scattering diagrams in the second row of Fig. 1 are finite, leading to an enhancemenet of the interaction terms that are proportional to $\hat{c}_{a}^{\dagger} \hat{c}_{b}^{\dagger} \hat{c}_{a} \hat{c}_{b}$. This factor of two enhancement can be thought of as being due to an exchange process; it is not specific to bosons and also exists in fermionic systems. The doubling of the mixed scattering diagrams for the lattice coupling case compared to the Raman coupling case leads - in certain parameter regimes - to distinct Rabi oscillations for scenarios 1 and 2. Good agreement between experimental and theoretical results is found and implications for, e.g., lattice calibration experiments are discussed.

Working in a parameter regime where the Rabi oscillations are noticeably impacted by the interactions, we probe the internal dynamics of condensed atom clouds through a Ramsey-type $\pi / 2-$ hold $-\pi / 2$ pulse sequence [13, which is of direct relevance to atom interferometry applications. Expanding upon earlier work [14[16, a theoretical framework is developed that explains the experimentally observed interference fringes. $\pi / 2$ pulses play an important role in momentum space engineering [17, 18]. For example, the splitting of an initial wave packet as well as the preparation of a variety of target states can be accomplished by $\pi / 2$ pulses. Perfect splitting may, however, be hampered by interactions and wave packet broadening. Intriguingly, combining Raman and lattice coupling schemes, which are considered separately in this paper, one might be able to realize loop-like plaquette systems. Specifically, the work presented in this paper provides a stepping stone for realizing a plaquette system, in which two momentum space lattices, occupied by two different hyperfine states, are coupled by Raman lasers.

The remainder of this article is organized as follows. Section II discusses the experimental setup for the Raman and lattice coupling schemes. Section III presents theory background and numerical results for the Raman coupling case. It also develops a fully analytical framework for the Ramsey-type pulse sequence. The agreement between the theoretical and experimental data for the Rabi oscillations is excellent. Section IV discusses the lattice coupling case; particular emphasis is placed on contrasting the dynamics for the lattice coupling scenario with that for the Raman coupling scenario. Experimental results are found to agree with the theoretical predictions quantitatively for the Rabi oscillation data and qualitatively for the Ramsey-type pulse-sequence data. Finally, Sec. V] summarizes our key findings and presents an outlook.

\section{EXPERIMENTAL SETUP}

The experiments are performed with a ${ }^{87} \mathrm{Rb} \mathrm{BEC}$ consisting of approximately $N=10^{5}$ atoms. Nearly pure BECs are confined in an elongated harmonic trap with trap frequencies of approximately $\left\{\omega_{x}, \omega_{y}, \omega_{z}\right\}=$ $2 \pi\{140,160,25\} \mathrm{Hz}$. The spin-independent trapping potential is produced by two crossed, optical dipole beams with a wavelength of $1064 \mathrm{~nm}$. Anharmonic corrections for this trapping configuration are estimated to be negligible for the purpose of this work. After preparation of the initial state, we remove the trapping potential at time $t=0$. For all practical purposes, the turning off of the external confinement is done instantaneously,

$$
V_{\text {trap }}(\mathbf{r}, t)=\left\{\begin{array}{ll}
\frac{m}{2}\left(\omega_{x}^{2} x^{2}+\omega_{y}^{2} y^{2}+\omega_{z}^{2} z^{2}\right) & \text { for } t<0 \\
0 & \text { for } t \geq 0
\end{array},\right.
$$

where $m$ denotes the atom mass. The trap frequencies and atom number are calibrated daily by fitting dipole oscillation data and cloud widths during expansion, respectively. The relevant values are reported in the figure captions.

Scenario 1 is realized by applying two $789.1 \mathrm{~nm}$ Raman lasers with effective coupling strength $\Omega_{R}$ and Raman detuning $\delta_{R}$ to couple the $\left|F, m_{F}\right\rangle=|1,-1\rangle=|\uparrow\rangle$ and $\left|F, m_{F}\right\rangle=|1,0\rangle=|\downarrow\rangle$ hyperfine states of ${ }^{87} \mathrm{Rb}$ under 
an external magnetic field of approximately $10 \mathrm{G}$. Here, $F$ denotes the total angular momentum of the ${ }^{87} \mathrm{Rb}$ atom and $m_{F}$ the corresponding projection quantum number. The two-photon Raman coupling scheme follows the procedure described in Ref. [19]. In momentum space, the two hyperfine states are separated by $2 \hbar k_{R}$, where $k_{R}$ is determined by the wave number and orientation of the Raman lasers. Specifically, the two Raman lasers with wave vectors $\mathbf{k}_{1}$ and $\mathbf{k}_{2}$ cross at an angle of $\theta_{R}$. Defining $2 k_{R}=\left|\mathbf{k}_{1}-\mathbf{k}_{2}\right|$ and using $\left|\mathbf{k}_{1}\right|=\left|\mathbf{k}_{2}\right|$, we have $k_{R}=\left|\mathbf{k}_{1}\right| \sin \left(\theta_{R} / 2\right)$; in our set-up, $\theta_{R} \approx \pi / 2$ or $k_{R} \approx\left|\mathbf{k}_{1}\right| / \sqrt{2}$. The difference between the angular frequencies $\omega_{1}$ and $\omega_{2}$ of the two lasers allows one to set the Raman detuning $\delta_{R}, \delta_{R}=4 E_{R}-\hbar \omega_{R}+E_{\text {Zeeman }}$, where $\omega_{R}=\omega_{1}-\omega_{2}$ and

$$
E_{R}=\frac{\hbar^{2} k_{R}^{2}}{2 m}
$$

Here, $E_{\text {Zeeman }}$ is the Zeeman splitting between the two coupled hyperfine states. The hyperfine state $|1,1\rangle$, which is off-resonant due to the quadratic Zeeman shift, is not included in our theoretical description. We have checked that inclusion of this state does not notably change the dynamics in the parameter regime of interest.

Scenario 2 is realized by preparing all atoms in the $\left|F, m_{F}\right\rangle=|1,-1\rangle=|\uparrow\rangle$ state and loading the BEC into a moving optical lattice [20]. Spin changing collisions play a negligible role in the magnetic fields used in this work. The lattice is created by crossing two $1064 \mathrm{~nm}$ lasers at an angle of $\theta_{L}\left(\theta_{L} \approx \pi / 2\right)$, with polarization perpendicular to the trapping beams, wave vectors $\mathbf{k}_{1}$ and $\mathbf{k}_{2}\left(\left|\mathbf{k}_{1}\right|=\left|\mathbf{k}_{2}\right|\right)$, and angular frequencies $\omega_{1}$ and $\omega_{2}$. The resulting lattice is characterized by the effective coupling strength $\Omega_{L}$, the wave vector $k_{L}$, and the detuning $\delta_{L}\left(k_{L} \approx\left|\mathbf{k}_{1}\right| / \sqrt{2}\right.$ and $\delta_{L}=4 E_{L}-\hbar \omega_{L}$, where $\left.\omega_{L}=\omega_{1}-\omega_{2}\right)$. Energies and lengths are measured in units of $E_{L}$ [Eq. (4) with the subscript " $R$ " replaced by " $L "]$ and $1 / k_{L}$, respectively. Specific values are given in the context of the experiments described below. In all cases, the external harmonic confinement is turned off time $t=0$.

In the remainder of this paper, we denote the coupling strength by $\Omega(t)$ when the discussion is independent of the specific scheme, i.e., when the discussion applies to both the Raman and lattice coupling cases. When the discussion is specific to one of the scenarios, we use, respectively, $\Omega_{R}(t)$ and $\Omega_{L}(t)$ for the Raman and lattice coupling cases [correspondingly, $\Omega_{0}$ in Eqs. (5) and (6) below are replaced by $\Omega_{0, R}$ and $\Omega_{0, L}$, respectively]. The coupling, which is assumed to be real, is turned on at time $t_{\text {start }}$, where $t=0$ is the time at which the trapping potential is removed. For $t_{\text {start }}>0$, the initial BEC expands in the absence of the Raman or lattice drive, thereby reducing the interaction strength during the subsequent pulse sequence. For the Rabi oscillation measurements, we keep $\Omega(t)$ on for a time interval

$$
t_{\text {seq }}=t_{\text {end }}-t_{\text {start }},
$$

$$
\Omega(t)= \begin{cases}0 & \text { for } t<t_{\text {start }} \\ \Omega_{0} & \text { for } t_{\text {start }} \leq t<t_{\text {end }} \\ 0 & \text { for } t \geq t_{\text {end }}\end{cases}
$$

For the Ramsey-type pulse sequence of length $t_{\text {seq }}=\tau_{1}+$ $t_{\text {hold }}+\tau_{2}$, the coupling strength $\Omega(t)$ reads

$$
\Omega(t)= \begin{cases}0 & \text { for } t<t_{\text {start }} \\ \Omega_{0} & \text { for } t_{\text {start }} \leq t<t_{\text {start }}+\tau_{1} \\ 0 & \text { for } t_{\text {start }}+\tau_{1} \leq t<t_{\text {start }}+\tau_{1}+t_{\text {hold }} \\ \Omega_{0} & \text { for } t_{\text {start }}+\tau_{1}+t_{\text {hold }} \leq t<t_{\text {end }} \\ 0 & \text { for } t \geq t_{\text {end }}\end{cases}
$$

In the experiment, the turning on of the coupling strength is not quite instantaneous but instead occurs over about $75 \mu \mathrm{s}$. To facilitate the comparison between theory and experiment, we choose $t_{\text {start }}$ to be the time at which $\Omega(t)$ has reached half of its maximum. In many applications that involve momentum transfer, a $\pi / 2$-wait$\pi$-wait- $\pi / 2$ pulse sequence is used instead of the shorter $\pi / 2$-wait- $\pi / 2$ pulse sequence. The reason we decided to apply the simpler pulse sequence is that the " $\pi$ reversal pulse" does not, as in other scenarios, remove the linear phase in our systems due to the presence of interactions.

The imaging is done at time $t_{\text {end }}+t_{\mathrm{ToF}}$, i.e., after an additional expansion time of $t_{\mathrm{ToF}}$. In the absence of the trapping potential, the momentum components separate naturally due to the fact that the states $\Psi_{a}$ and $\Psi_{b}$ have different velocities. For the lattice case, e.g., an expansion time of $t_{\mathrm{ToF}} \approx 10 \mathrm{~ms}$ corresponds to a separation of the cloud centers by about $85 \mu \mathrm{m}$ along the $z$-direction. This distance is larger than the size of the clouds after the expansion. For an initial cloud with Thomas-Fermi radius $22 \mu \mathrm{m}$, e.g., the size of the cloud at time $t_{\text {seq }}+t_{\text {ToF }}$ is about $43 \mu \mathrm{m}$.

Depending on the observable, the time-of-flight expansion plays no role, a negligible role, or an essential role when comparing experimental and theoretical data. For the Raman coupling case, the populations of the states $\Psi_{a}$ and $\Psi_{b}$ do not change during the time-of-flight expansion. This implies that theoretical results for the populations, calculated by neglecting the time-of-flight expansion, can be compared directly with experimentally measured populations. Correspondingly, we do not simulate the time-of-flight sequence when we compare Rabi oscillation data. Experiment-theory comparisons of the Ramsey-type pulse sequence, in contrast, require that the time-of-flight expansion be simulated to explain the observed fringe structures.

For the lattice case, the situation is slightly different. The populations of the states $\Psi_{a}$ and $\Psi_{b}$, which are distinguished only by their momentum, can change during the time-of-flight expansion due to atom-atom collisions that involve states with momenta $\approx n \hbar k_{L}$, where $n=-2, \pm 4, \pm 6, \cdots$. However, such population transfer is typically small; note that this is the reason why the two-state model introduced in Sec. IVA provides a 
reliable description for a fairly large parameter window. The small population transfer implies that the dynamics during the time-of-flight expansion can, in a first approximation, be neglected when analyzing populations. Understanding the internal dynamics such as the formation of density patterns, in contrast, requires that the time-of-flight sequence be modeled explicitly. which writes the mean-field spinor in terms of the components $\psi_{a}(\mathbf{r}, t)$ and $\psi_{b}(\mathbf{r}, t)$. Here and in what follows, $\psi_{a}$ and $\psi_{b}$ are time-dependent; note that the discussion in Sec. I adopted a stationary framework for simplicity. The unrotated $2 \times 2$ mean-field Hamiltonian $\hat{H}$ reads

\section{RAMAN COUPLING CASE}

\section{A. General framework}

Our theoretical analysis of the Raman-coupled system is based on the standard mean-field formulation [9],

$$
\begin{aligned}
& \hat{H}=\left(\frac{\hat{\mathbf{p}}^{2}}{2 m}+V_{\text {trap }}(\mathbf{r}, t)\right) \otimes I_{2}+\left(\begin{array}{cc}
g_{a a}\left|\psi_{a}(\mathbf{r}, t)\right|^{2}+g_{a b}\left|\psi_{b}(\mathbf{r}, t)\right|^{2} & 0 \\
0 & g_{b a}\left|\psi_{a}(\mathbf{r}, t)\right|^{2}+g_{b b}\left|\psi_{b}(\mathbf{r}, t)\right|^{2}
\end{array}\right)+ \\
& \left(\begin{array}{cc}
0 & \frac{\Omega_{R}(t)}{2} \exp \left(-2 \imath k_{R} z+\imath \omega_{R} t\right) \\
\frac{\Omega_{R}(t)}{2} \exp \left(2 \imath k_{R} z-\imath \omega_{R} t\right) & E_{\text {Zeeman }}
\end{array}\right)
\end{aligned}
$$

where $I_{2}$ is the $2 \times 2$ identity matrix and the normalization, expressed in terms of the fractional populations $N_{a}$ and $N_{b}$, is

$$
N_{a}+N_{b}=1
$$

with $(j=a$ or $b)$

$$
N_{j}=\int\left|\psi_{j}(\mathbf{r}, t)\right|^{2} d \mathbf{r} .
$$

The interaction strengths $g_{i j}$ between atoms in hyperfine states $i$ and $j$ are given by

$$
g_{i j}=\frac{4 \pi \hbar^{2}(N-1) a_{i j}}{m} .
$$

For ${ }^{87} \mathrm{Rb}$, we have $a_{a a}=100.4 a_{\mathrm{B}}, a_{a b}=a_{b a}=100.4 a_{\mathrm{B}}$, and $a_{b b}=100.9 a_{\mathrm{B}}$ 21, where $a_{\mathrm{B}}$ denotes the Bohr radius. In the arguments presented in Sec.I the four $g_{i j}$ were assumed to be the same; this simplifying assumption is again made in Sec. IIIE, The time dynamics of the system is governed by

$$
\imath \hbar \frac{\partial}{\partial t}\left(\begin{array}{c}
\psi_{a}(\mathbf{r}, t) \\
\psi_{b}(\mathbf{r}, t)
\end{array}\right)=\hat{H}\left(\begin{array}{c}
\psi_{a}(\mathbf{r}, t) \\
\psi_{b}(\mathbf{r}, t)
\end{array}\right) .
$$

Defining the rotated states $\tilde{\psi}_{a}(\mathbf{r}, t)$ and $\tilde{\psi}_{b}(\mathbf{r}, t)$,

$$
\left(\begin{array}{c}
\tilde{\psi}_{a}(\mathbf{r}, t) \\
\tilde{\psi}_{b}(\mathbf{r}, t)
\end{array}\right)=\hat{U}(z, t)\left(\begin{array}{c}
\psi_{a}(\mathbf{r}, t) \\
\psi_{b}(\mathbf{r}, t)
\end{array}\right),
$$

in terms of the rotation operator $\hat{U}(z, t)$,

$$
\hat{U}(z, t)=\left(\begin{array}{lc}
1 & 0 \\
0 & \exp \left(-2 \imath k_{R} z+\imath \omega_{R} t\right)
\end{array}\right),
$$

we obtain Eq. 11) with $\psi_{a}(\mathbf{r}, t), \psi_{b}(\mathbf{r}, t)$, and $\hat{H}$ replaced by $\tilde{\psi}_{a}(\mathbf{r}, t), \tilde{\psi}_{b}(\mathbf{r}, t)$, and $\hat{\tilde{H}}$, respectively, where the rotated Hamiltonian $\hat{\tilde{H}}$ is given by

$$
\begin{aligned}
\hat{\tilde{H}}= & \left(\frac{\hat{\mathbf{p}}^{2}}{2 m}+V_{\text {trap }}(\mathbf{r}, t)\right) \otimes I_{2}+\left(\begin{array}{cc}
g_{a a}\left|\tilde{\psi}_{a}(\mathbf{r}, t)\right|^{2}+g_{a b}\left|\tilde{\psi}_{b}(\mathbf{r}, t)\right|^{2} & 0 \\
0 & g_{b a}\left|\tilde{\psi}_{a}(\mathbf{r}, t)\right|^{2}+g_{b b}\left|\tilde{\psi}_{b}(\mathbf{r}, t)\right|^{2}
\end{array}\right)+ \\
& \left(\begin{array}{cc}
0 & \frac{\Omega_{R}(t)}{2} \\
\frac{\Omega_{R}(t)}{2} & \frac{2 \hbar k_{R} \hat{p}_{z}}{m}+\delta_{R}
\end{array}\right)
\end{aligned}
$$

To obtain Eq. (14), we used the relation $\left|\tilde{\psi}_{j}(\mathbf{r}, t)\right|^{2}=\left|\psi_{j}(\mathbf{r}, t)\right|^{2}$, where $j=a$ or $b$. Importantly, the position- 
and time-dependent phase $\tilde{\gamma}_{b}(\mathbf{r}, t)$ of the rotated component $\tilde{\psi}_{b}(\mathbf{r}, t)$ differs from the phase $\gamma_{b}(\mathbf{r}, t)$ of the unrotated component $\psi_{b}(\mathbf{r}, t)$. Since the change of the phases of the unrotated spinor components is dominated by the laser coupling term, thereby masking the change due to the internal dynamics, it is more convenient to analyze the phases of the spinor-components in the rotated basis, whose phase dynamics is governed by "internal effects" as opposed to the laser coupling.

For the Rabi oscillation measurements and the Ramsey-type pulse sequence, the BEC is initially (i.e., at $t=0)$ prepared in the state $\psi_{a}(\mathbf{r}, t)|\uparrow\rangle=\tilde{\psi}_{a}(\mathbf{r}, t)|\uparrow\rangle$, which is characterized by a vanishing average mechanical momentum along the $z$-direction, i.e., $\left\langle\hat{p}_{z}\right\rangle_{\text {initial }}=0$. Our calculations assume an axially symmetric harmonic trap with $\omega_{x}=\omega_{y}=\omega_{\rho}$. The trapping potential defines the harmonic oscillator lengths $a_{\mathrm{ho}, z}$ and $a_{\mathrm{ho}, \rho}$,

$$
a_{\mathrm{ho}, z / \rho}=\sqrt{\frac{\hbar}{m \omega_{z / \rho}}} .
$$

The coupled mean-field equations are solved using standard techniques. The initial state is obtained by imaginary time propagation. The real time dynamics is implemented by expanding the time evolution operator in terms of Chebychev polynomials [22, 23. We use equally spaced grid points in $z$ and $\rho$. The convergence of the results presented has been tested with respect to the size of the simulation box, the number of grid points, and the time step.

\section{B. Rabi oscillations: Vanishing Raman detuning}

This section discusses Rabi oscillation results for the Raman coupling case with $\delta_{R}=0$. The numerical solutions are obtained by solving the time-dependent meanfield equation for $\hat{\tilde{H}}$ [see Eq. 14 ] ] with $E_{R} / h=1960 \mathrm{~Hz}$. The Raman coupling is turned on at $t=0$, i.e., we have $t_{\text {start }}=0$. Figs. 2(a)-2(c) show the difference $N_{a}-N_{b}$ between the fractional populations as a function of the dimensionless time $t_{\mathrm{seq}} \Omega_{0, R} / h$ for different $N, \omega_{z}$, and $\Omega_{0, R}$, respectively.

Figure 2(a) shows numerical results for $\Omega_{0, R}=E_{R}$ and three different $N$, namely $N=1,3 \times 10^{5}$, and $N=10^{6}$. Even though the Rabi coupling lasers are turned on, at time $t=0$, after the trapping potential has been switched off, the figure caption quotes the trapping frequencies since they determine the initial state and thus the distribution of the kinetic and potential energy, including the mean-field energy, in the system. For the non-interacting single-atom system [black solid line in Fig. 2(a)], the Rabi oscillation period is nearly constant for the times considered; the amplitude, however, is visibly damped. While this "non-perfect" sinusoidal behavior might be surprising at first sight, it can be explained as follows: The center of mass of the component $\tilde{\psi}_{b}(\mathbf{r}, t)$ moves relative

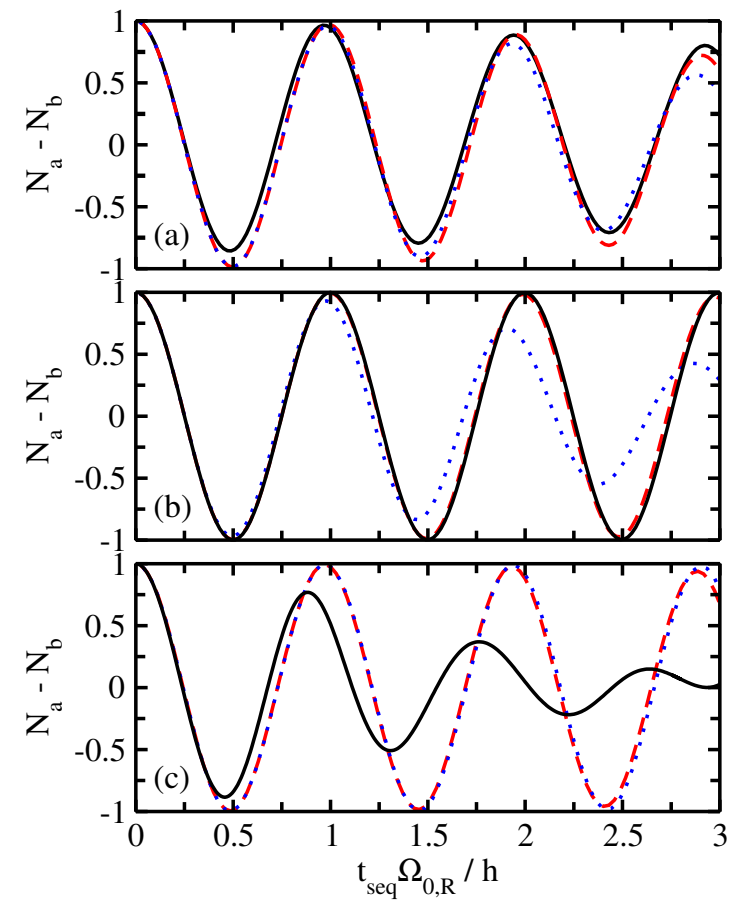

FIG. 2: Rabi oscillations for Raman coupling case (numerical results). The lines show the difference $N_{a}-N_{b}$ between the fractional populations as a function of the dimensionless time $t_{\text {seq }} \Omega_{0, R} / h$ for $t_{\text {start }}=0, E_{R} / h=1960 \mathrm{~Hz}, \delta_{R}=0$, and $\omega_{\rho}=2 \pi \times 200 \mathrm{~Hz}$. (a) Changing the particle number $N$. The black solid, red dashed, and blue dotted lines are for $N=1$, $N=3 \times 10^{5}$, and $N=10^{6}$, respectively. The weak trapping frequency is $\omega_{z}=2 \pi \times 40 \mathrm{~Hz}$ and the coupling strength is $\Omega_{0, R}=E_{R}$. (b) Changing the angular trapping frequency $\omega_{z}$. The black solid, red dashed, and blue dotted lines are for $\omega_{z}=2 \pi \times 10 \mathrm{~Hz}, \omega_{z}=2 \pi \times 20 \mathrm{~Hz}$, and $\omega_{z}=2 \pi \times 60 \mathrm{~Hz}$, respectively. The coupling strength is $\Omega_{0, R}=E_{R}$ and the number of particles is $N=3 \times 10^{5}$. (c) Changing the coupling strength $\Omega_{0, R}$. The black solid, red dashed, and blue dotted lines are for $\Omega_{0, R}=E_{R} / 2, \Omega_{0, R}=3 E_{R} / 2$, and $\Omega_{0, R}=5 E_{R} / 2$, respectively. The number of particles is $N=3 \times 10^{5}$ and the weak trapping frequency is $\omega_{z}=2 \pi \times 40 \mathrm{~Hz}$.

to the center of mass of the component $\tilde{\psi}_{a}(\mathbf{r}, t)$ during the Rabi oscillations. Thus, the two components are not perfectly overlapping spatially. As a consequence, the relative phase of the spinor components at fixed $\mathbf{r}$ is changing slightly due to the relative motion of the two components with respect to each other. This phase difference is responsible for the non-perfect population transfer ("damping"). An alternative but equivalent picture is that the finite momentum width of the initial state corresponds to a small effective momentum-dependent detuning. This effective detuning decreases with increasing mean-field interactions due to the decrease of the width of the initial state in momentum space.

When mean-field interactions are present [the red dashed and blue dotted lines in Fig. 2(a) are for $N=$ $3 \times 10^{5}$ and $N=10^{6}$, respectively], the amplitude of the Rabi oscillation data changes somewhat while the oscil- 
lation period is essentially unaffected by the interactions. For these two $N$-values, the chemical potential (in units of $h$ ) of the initial state is $\approx 3303 \mathrm{~Hz}$ and $5347 \mathrm{~Hz}$ (corresponding to $1.685 E_{R}$ and $2.728 E_{R}$ ), i.e., the chemical potential at $t=0$ is larger than $\Omega_{0, R}$.

To highlight the effect of the interactions, Fig. 2(b) shows the Raman-induced Rabi oscillations for $N=3 \times$ $10^{5}$ [same atom number as used for the red dashed line in Fig. 2(a)] for weaker and stronger confinement along the $z$-direction than used in Fig. 2(a). Stronger confinement leads to higher density and thus to enhanced interaction effects. For the largest $\omega_{z}$ considered [blue dotted line in Fig. 2(b)], the fractional population difference $N_{a}-$ $N_{b}$ deviates appreciably from a simple sinusoidal curve after a few oscillations. This indicates that care needs to be taken when calibrating the effective Raman coupling strength $\Omega_{0, R}$; in particular, a fit to a simple sinusoidal function, applicable to the ideal two-level model, might not yield the correct effective coupling strength.

The results presented in Figs. 2(a)-2(b) are for $\Omega_{0, R}=$ $E_{R}$. Figure 2(c) shows the dynamics for smaller and larger coupling strengths, namely $\Omega_{0, R}=E_{R} / 2, \Omega_{0, R}=$ $3 E_{R} / 2, \Omega_{0, R}=5 E_{R} / 2$, and the same trap confinement as in Fig. 2(a). Even though the particle number is quite moderate (namely, $N=3 \times 10^{5}$ ), the oscillation amplitude and period for $\Omega_{0, R}=E_{R} / 2$ [solid line in Fig. 2(c)] deviate strongly from perfect sinusoidal behavior due to the enhanced effects of the mean-field energy with decreasing $\Omega_{0, R}$. This implies that care needs to be exercised if the calibration of the effective Raman coupling strength is done for low coupling strengths. In this regime, one has to make sure that the particle number is sufficiently low or that one allows for sufficient timeof-flight expansion prior to turning on the Raman Rabi coupling (Fig. 2 is for $t_{\text {start }}=0$ ). If this is not done, the value of the effective Rabi coupling strength, which enters into the underlying system Hamiltonian, may be impacted by interaction effects, potentially leading to errors in experiments that require reliable precision, such as quantum analog simulations and many-body studies. Alternatively, explicit comparisons with Gross-Pitaevskii equation results, as done in this work, would be very useful when interactions are present. Last, one may consider performing the calibration in the "large power" regime and extrapolating the resulting calibration curve instead of performing the calibration in the "low power" regime.

\section{Rabi oscillations: Theory-experiment comparison}

Figure 3 shows a comparison between theory and experiment for the Raman-induced Rabi oscillations for an initial state with chemical potential $\mu=0.8436 E_{R}$. This chemical potential corresponds to a mean-field energy per particle at $t=0$ of $0.5206 E_{R}$. To reduce the mean-field energy in the system, a $0.5 \mathrm{~ms}$ free expansion step was inserted after tuning off the trap and prior to turning on the Raman coupling lasers. At the end of the free expansion, the mean-field energy per particle is about $20 \%$ smaller than at $t=0$. The resulting Rabi oscillations are slightly damped. Although the experimental data (red dots) are obtained for a small negative detuning $\delta_{R}$, the detuning is not the only cause for the damping. Extrapolating the mean-field Gross-Pitaevskii results for finite detuning to zero detuning, we conclude that even the zero detuning case displays damping [explicit calculations for $\delta_{R}=0$ (not shown) confirm this]. Combining the good agreement between the experimental data and theoretical curves with the discussion of the previous section, we conclude that the damping can be partially attributed to the mean-field interactions. Indeed, if we let the BEC expand longer prior to turning on the Raman coupling, the damping or dephasing, for the same detuning $\delta_{R}$, is reduced.

Interestingly, fitting of the mean-field Gross-Pitaevskii results for $\delta_{R} / h=-200 \mathrm{~Hz}$ (this detuning gives the best agreement with the experimental data) to a damped periodic function of the form

$$
N_{a}-N_{b}=\cos (2 \pi f t) \exp (-t / \tau)
$$

yields a frequency $f$ of $2578 \mathrm{~Hz}$. This "fitted Rabi coupling strength" is about $1.2 \%$ larger than the Rabi coupling strength $\Omega_{0, R}$ used in the simulations. This indicates that the interactions impact, for the parameter combinations considered, the oscillation frequency much less than the amplitude. More specifically, for the parameter combination considered in Fig. 3 , the effect of the interactions on the Rabi oscillations can be described, to a good approximation, phenomenologically by the time constant $\tau$.

\section{Ramsey-type pulse sequence: Theory overview}

Throughout this section, the initial BEC $\left(N=3 \times 10^{5}\right)$ is prepared for a confinement with $\omega_{\rho}=2 \pi \times 200 \mathrm{~Hz}$ and $\omega_{z}=2 \pi \times 40 \mathrm{~Hz}$. The dynamics are analyzed for the Ramsey-type pulse sequence with Raman coupling strength $\Omega_{0, R}=E_{R}$, detuning $\delta_{R}=0$, and-as in Sec. IIIB $-E_{R} / h=1960 \mathrm{~Hz}$ and $t_{\text {start }}=0$. The main emphasis lies on developing, motivated by numerical simulations of the time-dependent mean-field equation for the Hamiltonian given in Eq. (14), a benchmark and physical picture that provides the motivation for the analytical treatment presented in Sec. IIIE.

When the Raman coupling is turned on, population is transferred from the component $\tilde{\psi}_{a}(\mathbf{r}, t)$ to the component $\tilde{\psi}_{b}(\mathbf{r}, t)$. As discussed in the previous sections, the interactions can notably impact the Rabi oscillations, in particular for longer times. Despite of this, we measure the lengths of our pulses in terms of the characteristic time scale of the non-interacting system, i.e., we refer to a $\pi / 2$-pulse as a pulse that transfers, in the absence of interactions and assuming an infinitely narrow momentum space wave packet, half of the atoms from 


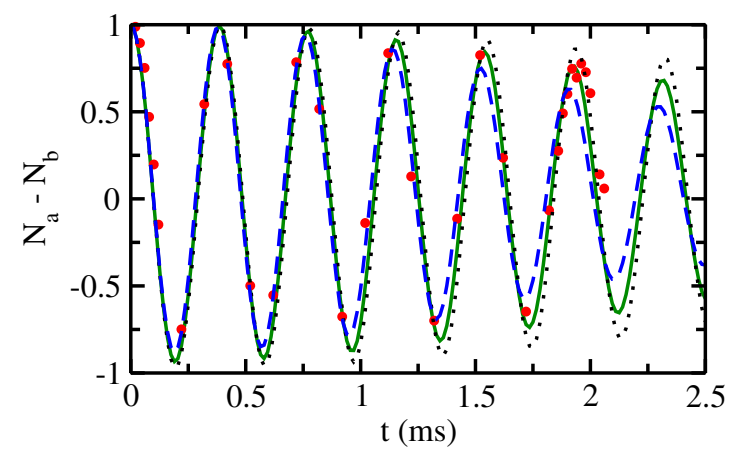

FIG. 3: Theory-experiment comparison for Raman Rabi oscillations for a ${ }^{87} \mathrm{Rb}$ BEC. The red dots show the experimentally determined difference in the fractional populations as a function of time. The experimental parameters are $\omega_{x}=2 \pi \times 155 \mathrm{~Hz}, \omega_{y}=2 \pi \times 179 \mathrm{~Hz}, \omega_{z}=2 \pi \times 24.8 \mathrm{~Hz}$, $N=1.5 \times 10^{5}, E_{R} / h=1960 \mathrm{~Hz}, \Omega_{0, R} / h=2548 \mathrm{~Hz}$, and $t_{\text {start }}=0.5 \mathrm{~ms}$. The experiments were performed for a small negative detuning $\delta_{R} / h$ that is estimated to be between $-200 \mathrm{~Hz}$ and $-600 \mathrm{~Hz}$, where the uncertainty is due to fluctuations in the external magnetic field responsible for the Zeeman splitting. The solutions to the mean-field GrossPitaevskii equation (lines) are obtained for an axially symmetric trap characterized by the experimentally measured $\omega_{z}$ and $\omega_{\rho}=2 \pi \times 167.0 \mathrm{~Hz}$ ( $\omega_{\rho}$ is taken to be the mean of the experimental $\omega_{x}$ and $\left.\omega_{y}\right)$. The black dotted, green solid, and blue dashed lines show results for $\delta_{R} / h=-200 \mathrm{~Hz},-400 \mathrm{~Hz}$, and $-600 \mathrm{~Hz}$, respectively; the other parameters are taken from experiment. The results for $\delta_{R} / h=-200 \mathrm{~Hz}$ describe the experimental data the best. The chemical potential $\mu$ prior to turning off the trap is $0.8436 E_{R}$. The mean-field energy per particle prior to turning off the trap and after the $0.5 \mathrm{~ms}$ expansion is $0.5206 E_{R}$ and $0.4076 E_{R}$, respectively.

the state $\tilde{\psi}_{a}(\mathbf{r}, t)$ to the state $\tilde{\psi}_{b}(\mathbf{r}, t)$. For the parameters employed in Fig. 4, a $\pi / 2$-pulse corresponds to $\pi h /\left(2 \Omega_{0, R}\right) \approx 0.1276 \mathrm{~ms}$. Figure 4 (ai) shows that the population of state $\tilde{\psi}_{b}(\mathbf{r}, t)$ after the first $\pi / 2$-pulse is close to $50 \%$ (it is $49.95 \%$ ). In addition, it can be seen that the population in the component $\tilde{\psi}_{b}(\mathbf{r}, t)$ moves a tiny bit relative to the population in the component $\tilde{\psi}_{a}(\mathbf{r}, t)$ during the first $\pi / 2$-pulse. The reason is that the population in state $\tilde{\psi}_{b}(\mathbf{r}, t)$ has an average mechanical momentum of about $2 \hbar k_{R}$ along the $z$-direction while the population in state $\tilde{\psi}_{a}(\mathbf{r}, t)$ has an average mechanical momentum very close to zero along the $z$-direction.

During the variable hold time, no population transfer occurs since the Raman coupling lasers are turned off. The two key characteristics during the hold time are: First, the population in state $\tilde{\psi}_{b}(\mathbf{r}, t)$ continues to move relative to that in component $\tilde{\psi}_{a}(\mathbf{r}, t)$. Second, the interacting BEC expands a tiny bit. Figures 4 (aii) and 4(aiii) show $\rho=0$ cuts and are for $t_{\text {hold }}=1 \mathrm{~ms}$ and $t_{\text {hold }}=2 \mathrm{~ms}$, respectively.

To "reunite" the populations of the states $\tilde{\psi}_{a}(\mathbf{r}, t)$ and $\tilde{\psi}_{b}(\mathbf{r}, t)$, a second $\pi / 2$-pulse is applied. For the example shown in Fig. 4 , the population of state $\tilde{\psi}_{a}(\mathbf{r}, t)$ after the second $\pi / 2$-pulse is $51.74 \%$ for $t_{\text {hold }}=1 \mathrm{~ms}$ and $50.15 \%$ for $t_{\text {hold }}=2 \mathrm{~ms}$. After the second $\pi / 2$-pulse, the density profiles [see Figs. 4(aiv)-4(av) for $\rho=0$ cuts] show interference fringes in the "central" or "overlap" region, i.e., in the spatial region where the two clouds overlapped prior to the application of the second $\pi / 2$-pulse. The interference fringes establish themselves through out-ofphase oscillations of $\left|\tilde{\psi}_{a}(z, 0)\right|^{2}$ and $\left|\tilde{\psi}_{b}(z, 0)\right|^{2}$. We observe analogous fringes for other $\rho$ values. The total density of the two components (not shown), in contrast, exhibits oscillations with comparatively small amplitude in the outer region and no oscillations in the central region.

Quite generally, the appearance of fringes such as those displayed in Figs. 4(aiv) and 4(av) suggests the existence of two interfering pathways, i.e., the existence of a spatially dependent phase difference. In the following, we introduce a theoretical framework that highlights how the fringe pattern develops for the Ramsey-type pulse sequence with Raman coupling. To this end, it is instructive to visualize the time-evolving rotated spinor on the Bloch sphere. Since the two components $\tilde{\psi}_{a}(\mathbf{r}, t)$ and $\tilde{\psi}_{b}(\mathbf{r}, t)$ can each be written in terms of one complex number for each $z, \rho$, and $t$ (the axial symmetry suggests the use of cylindrical coordinates), we define

$$
\begin{aligned}
&\left(\begin{array}{c}
\tilde{\psi}_{a}(z, \rho, t) \\
\tilde{\psi}_{b}(z, \rho, t)
\end{array}\right)=R(z, \rho, t) \exp \left[\imath \tilde{\gamma}_{a}(z, \rho, t)\right] \times \\
&\left(\begin{array}{c}
\cos \left(\frac{\theta(z, \rho, t)}{2}\right) \\
\exp [\imath \phi(z, \rho, t)] \sin \left(\frac{\theta(z, \rho, t)}{2}\right)
\end{array}\right),
\end{aligned}
$$

where

$$
\begin{gathered}
R(z, \rho, t)=\sqrt{\left|\tilde{\psi}_{a}(z, \rho, t)\right|^{2}+\left|\tilde{\psi}_{b}(z, \rho, t)\right|^{2}}, \\
\theta(z, \rho, t)=2 \arctan \left(\frac{\left|\tilde{\psi}_{b}(z, \rho, t)\right|}{\left|\tilde{\psi}_{a}(z, \rho, t)\right|}\right),
\end{gathered}
$$

and

$$
\phi(z, \rho, t)=\tilde{\gamma}_{b}(z, \rho, t)-\tilde{\gamma}_{a}(z, \rho, t) .
$$

Here, $\tilde{\gamma}_{a}(z, \rho, t)$ can be interpreted as an overall spatially dependent phase of the spinor wave function. This phase has no effect on the physical observables considered in this work. The quantity $R(z, \rho, t)$ corresponds to a "weight" at each spatial point. The spinor dynamics for a given $z$ and $\rho$ is thus conveniently visualized by a vector of length $R(z, \rho, t)$ on the Bloch sphere. The direction of the vector is given by $\theta(z, \rho, t)$ and the relative phase $\phi(z, \rho, t)$ between the components $\tilde{\psi}_{b}(z, \rho, t)$ and $\tilde{\psi}_{a}(z, \rho, t)$.

To visualize the motion of the spinor on the Bloch sphere, we define the local spin expectation values $\sigma_{j}(z, \rho, t)$, where $j=x, y$, or $z$, through

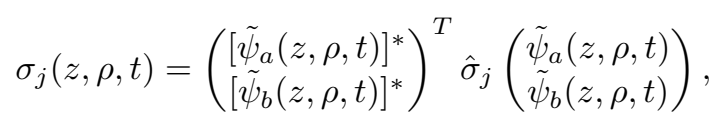




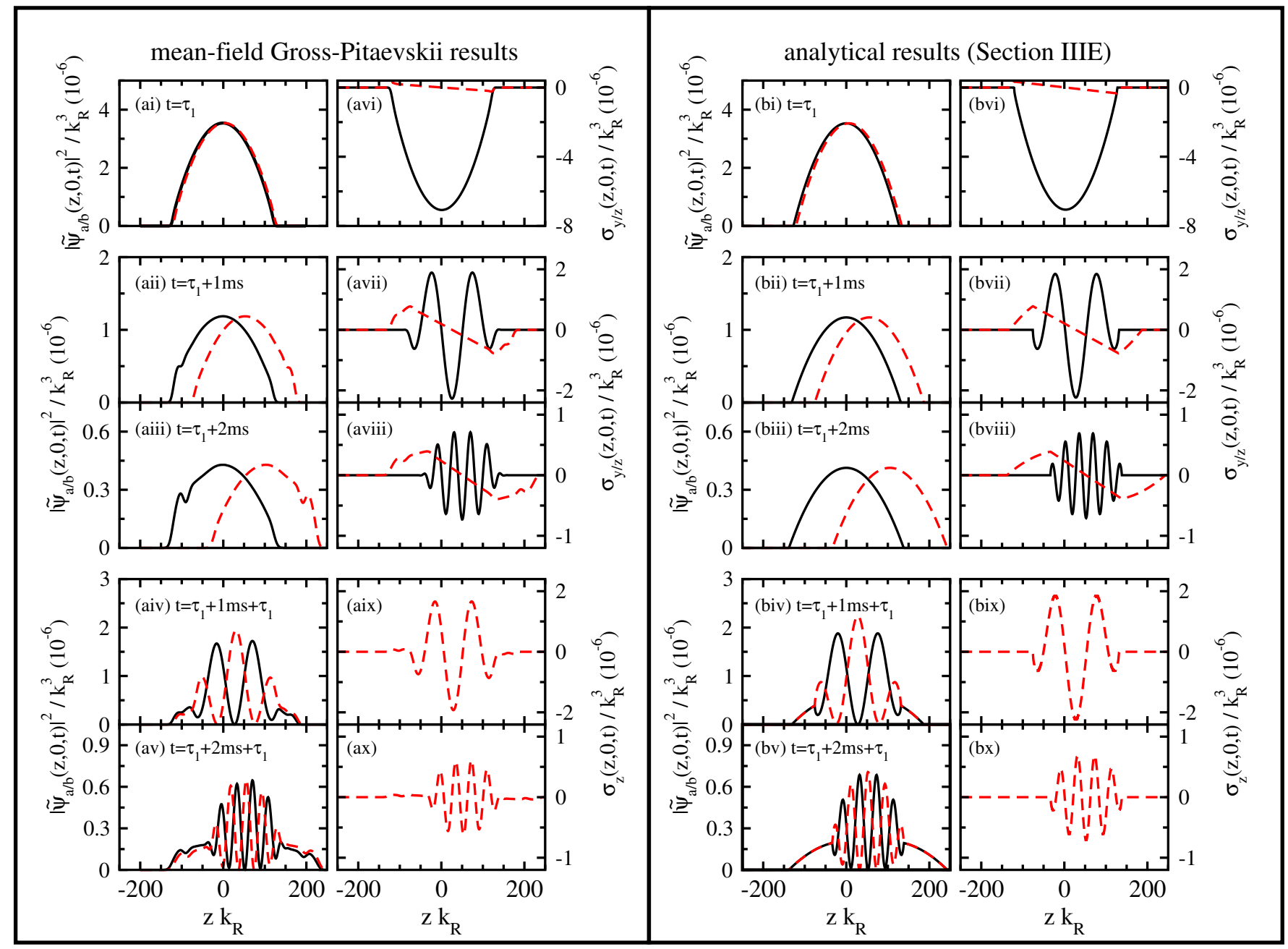

FIG. 4: Density cuts and local spin expectation values for the Ramsey-type pulse sequence with $E_{R} / h=1960 \mathrm{~Hz}, \Omega_{0, R}=E_{R}$, and $\delta_{R}=0$ (theory results). The ${ }^{87} \mathrm{Rb}$ BEC consists of $N=3 \times 10^{5}$ atoms and is prepared in an axially symmetric trap with $\omega_{\rho}=2 \pi \times 200 \mathrm{~Hz}$ and $\omega_{z}=2 \pi \times 40 \mathrm{~Hz}$. This corresponds to a chemical potential, in units of $h$, of $\approx 3303 \mathrm{~Hz}$. All results are obtained for $t_{\text {start }}=0$. The first and second columns are obtained by solving the time-dependent mean-field equation for the Hamiltonian given in Eq. 14 numerically. The third and fourth columns show the same observables as the first and second columns but are, instead, calculated using the fully analytical framework developed in Sec. III E the agreement is very good. The black solid and red dashed lines in panels (ai)-(av) show the density cuts $\left|\tilde{\psi}_{a}(z, 0, t)\right|^{2}$ and $\left|\psi_{b}(z, 0, t)\right|^{2}$, respectively. The black solid and red dashed lines in panels (avi)-(ax) show the local spin expectation values $\sigma_{y}(z, 0, t)$ and $\sigma_{z}(z, 0, t)$, respectively. The time increases from the first row, to the second/third row, to the fourth/fifth row (the value of the time is given in the panels); the second and fourth row correspond to a hold time of $1 \mathrm{~ms}$, and the third and fifth row correspond to a hold time of $2 \mathrm{~ms}$. It can be seen that the agreement between the mean-field Gross-Pitaevskii results and the fully analytical results is quite good. The figure illustrates that the second $\pi / 2$-pulse transfers the information encoded in $\sigma_{y}(z, 0, t)$ to $\sigma_{z}(z, 0, t)$, making the interference visible in the population difference.

where $\hat{\sigma}_{x}, \hat{\sigma}_{y}$, and $\hat{\sigma}_{z}$ denote the "usual" Pauli matrices. Note that we use the term "spin expectation value" for convenience throughout this paper even though our definition in Eq. (21) excludes the conventional $\hbar / 2$ factor. Physically, $\sigma_{z}(z, \rho, t)$ corresponds to the local $(z, \rho)$ specific population difference at time $t$. Mathematically, one finds

$$
\begin{aligned}
& \sigma_{x}(z, \rho, t)=|R(z, \rho, t)|^{2} \cos (\phi(z, \rho, t)) \sin (\theta(z, \rho, t)), \\
& \sigma_{y}(z, \rho, t)=|R(z, \rho, t)|^{2} \sin (\phi(z, \rho, t)) \sin (\theta(z, \rho, t)),
\end{aligned}
$$

and

$$
\sigma_{z}(z, \rho, t)=|R(z, \rho, t)|^{2} \cos (\theta(z, \rho, t)) .
$$

The initial state at $t=0$ corresponds to a vector pointing to the north pole on the Bloch sphere. From the Hamiltonian $\hat{\tilde{H}}$ [Eq. [14]], it can be seen that the non-vanishing Raman coupling term introduces a torque along the positive $x$-axis. Thus, neglecting interactions, the first $\pi / 2$-pulse rotates the spinor wave function by $-\pi / 2$ about the $x$-axis on the Bloch sphere. As a re- 
sult, the spinor points along the negative $y$-axis on the Bloch sphere after the first $\pi / 2$-pulse. Our numerical mean-field results, which show that $\theta$ and $\phi$ are approximately equal to $\pi / 2$ and $-\pi / 2$ across the entire BEC after the first $\pi / 2$-pulse are consistent with this simple picture. Correspondingly, $\sigma_{z}(z, 0, t)$ is approximately zero and $\sigma_{y}(z, 0, t)$ has - except for a minus sign - the same $z$-dependence as the density [red dashed and black solid lines Fig. 4(avi)]. We conclude that mean-field effects can, in a first-order approximation, be neglected during the first $\pi / 2$-pulse.

During the hold time, two effects need to be accounted for: First, as already pointed out earlier, the population in state $\tilde{\psi}_{b}(\mathbf{r}, t)$ moves relative to that in state $\tilde{\psi}_{a}(\mathbf{r}, t)$. Second, the phases of the spinor components $\tilde{\psi}_{a}(z, \rho, t)$ and $\tilde{\psi}_{b}(z, \rho, t)$ evolve independently. For each $(z, \rho)$, the combination of these two effects leads to a rotation of the two-component spinor on the Bloch sphere. As an example, Figs. 4(avii)-4(aviii) show $\sigma_{z}(z, 0, t)$ and $\sigma_{y}(z, 0, t)$ for two different hold times. For both hold times, $\sigma_{z}(z, 0, t)$ changes approximately linearly with $z$ in the region where the two components overlap. This follows immediately from the approximately parabolic shapes of the two density components, which are offset from each other: the difference leads to a term that is, to leading order, linear in $z$. The local spin expectation value $\sigma_{y}(z, 0, t)$ develops "wiggles" during the hold time in the region where the two components overlap. The number of wiggles increases with increasing hold time. The wiggles arise from the relative phase dynamics and indicate interference; importantly, the densities do not show any indication of interference prior to the application of the second $\pi / 2$-pulse.

The second $\pi / 2$-pulse "rotates" $\sigma_{y}(z, \rho, t)$ to $\sigma_{z}(z, \rho, t)$. This can be seen clearly by comparing Fig. 4(aix) with Fig. 4(avii) (both these figures are for a hold time of $1 \mathrm{~ms}$ ) and by comparing Fig. 4(ax) with Fig. 4(aviii) (both these figures are for a hold time of $2 \mathrm{~ms}$ ). Since the relative phase information has been "moved" from $\sigma_{y}(z, \rho, t)$ to $\sigma_{z}(z, \rho, t)$ and since $\sigma_{z}(z, \rho, t)$ is equal to $\left|\tilde{\psi}_{a}(z, \rho, t)\right|^{2}-\left|\tilde{\psi}_{b}(z, \rho, t)\right|^{2}$, the interference is - after the second $\pi / 2$-pulse - visible in the densities of the components [Figs. 4(aiv)-4(av)].

\section{E. Ramsey-type pulse sequence: Analytical treatment}

The numerical results presented in the previous section are obtained using the scattering lengths $a_{i j}$ for ${ }^{87} \mathrm{Rb}$. Repeating the numerical calculations for equal scattering lengths $a_{i j}$ reveals that the effects due to the difference in the scattering lengths are quite small for the time scales considered in this paper. Motivated by this observation, the analytical treatment presented in this section makes the simplifying assumption that the scattering lengths are all equal $\left(a_{a a}=a_{a b}=a_{b a}=a_{b b}\right)$. Moreover, the treatment assumes that $\delta_{R}$ and $t_{\text {start }}$ are equal to zero.
Our analytical model is motivated by Refs. 14, 15; however, the application to the Ramsey-type pulse sequence discussed here has, to the best of our knowledge, not been discussed previously.

We assume that the initial state $\tilde{\psi}_{a}(\mathbf{r}, t=0)$ is described well within the Thomas-Fermi approximation. We additionally assume that the population transfer process commutes with the relative moving and expansion processes during the first $\pi / 2$-pulse. Specifically, our analytical model treats the population transfer associated with the first $\pi / 2$-pulse as occuring instantaneously and then subsequently treats the relative moving and expansion of the two components for the duration $\tau_{1}$ of the actual $\pi / 2$-pulse. For the second $\pi / 2$-pulse, we reverse the order of the operations, i.e., we first treat the relative moving and expansion of the two components for the duration $\tau_{2}$ and then treat the population transfer associated with the second $\pi / 2$-pulse as occuring instantaneously. In the following, we provide an analytical framework that yields approximate expressions for $\tilde{\psi}_{a}(z, \rho, t)$ and $\tilde{\psi}_{b}(z, \rho, t)$ right after the first instantaneous $\pi / 2$ pulse $\left(t=0^{+}\right)$, during the time $0^{+}<t<\tau_{1}+t_{\text {hold }}+\tau_{2}$, and right after the second instantaneous $\pi / 2$-pulse $(t=$ $\left.t_{\text {end }}^{+}\right)$.

Motivated by the discussion in Sec. IIIB we make the ansatz that $\left|\tilde{\psi}_{a}(\mathbf{r}, t)\right|^{2}$ has an inverted parabola-like form during the "effective" hold time, i.e., for $0^{+}<t<t_{\text {end }}$,

$$
\frac{1}{\lambda_{z}(t) \lambda_{\rho}^{2}(t)}\left[-\alpha_{z}\left(\frac{z}{\lambda_{z}(t)}\right)^{2}-\alpha_{\rho}\left(\frac{\rho}{\left.(\mathbf{r}, t)\right|^{2}}=\right.\right.
$$

where $\mu$ denotes the chemical potential of the initial state, i.e., of the system prior to the application of the first $\pi / 2$-pulse [24,

$$
\mu=\frac{1}{2}\left[\omega_{\rho}^{4} \omega_{z}^{2} m^{3}\left(\frac{15 g}{4 \pi}\right)^{2}\right]^{1 / 5},
$$

and

$$
\alpha_{z / \rho}=\frac{m \omega_{z / \rho}^{2}}{4 g} .
$$

Compared to the "standard case" [14], $\alpha_{\rho}$ and $\alpha_{z}$ are smaller by a factor of 2 since the population for $t=0^{+}$is assumed to be equally distributed between the two components, i.e., $\left|\tilde{\psi}_{b}\left(\mathbf{r}, 0^{+}\right)\right|=\left|\tilde{\psi}_{a}\left(\mathbf{r}, 0^{+}\right)\right|$. In Eq. 25$)$, it is understood that $\left|\tilde{\psi}_{a}(\mathbf{r}, t)\right|$ is zero when the right hand side of the equation takes negative values. The dimensionless scaling parameters $\lambda_{z}(t)$ and $\lambda_{\rho}(t)$ obey the initial conditions $\lambda_{z}\left(0^{+}\right)=\lambda_{\rho}\left(0^{+}\right)=1$. The differential equations that govern the time evolution of $\lambda_{z}(t)$ and $\lambda_{\rho}(t)$ are discussed below. We set $\tilde{\gamma}_{a}\left(\mathbf{r}, 0^{+}\right)=0$ and assume that the first $\pi / 2$-pulse introduces a $-\pi / 2$ phase shift onto the second component, i.e., $\tilde{\gamma}_{b}\left(\mathbf{r}, 0^{+}\right)=-\pi / 2$. 
For $0^{+}<t<t_{\text {end }}, \tilde{\psi}_{b}(\mathbf{r}, t)$ moves with approximately constant velocity $v_{z}$,

$$
v_{z}=\frac{2 \hbar k_{R}}{m},
$$

along the $z$-direction while the center-of-mass of $\left|\tilde{\psi}_{a}(\mathbf{r}, t)\right|^{2}$ remains essentially unchanged. Due to the symmetry of the system, we enforce

$$
\tilde{\psi}_{b}(\mathbf{r}, t)=\tilde{\psi}_{a}\left(v_{z} t \hat{e}_{z}-\mathbf{r}, t\right) \exp \left(-\imath \frac{\pi}{2}\right) .
$$

Thus, once we have expressions for $\left|\tilde{\psi}_{a}\left(v_{z} t \hat{e}_{z}-\mathbf{r}, t\right)\right|$ and $\tilde{\gamma}_{a}\left(v_{z} t \hat{e}_{z}-\mathbf{r}, t\right), \tilde{\psi}_{b}(\mathbf{r}, t)$ is determined through Eq. 29. To eliminate $\tilde{\psi}_{b}(\mathbf{r}, t)$, we insert Eq. 29 into the coupled set of time-dependent mean-field equations. This yields

$$
\imath \hbar \frac{\partial}{\partial t} \tilde{\psi}_{a}(\mathbf{r}, t)=\hat{\tilde{H}}_{\mathrm{hold}} \tilde{\psi}_{a}(\mathbf{r}, t),
$$

where

$$
\hat{\tilde{H}}_{\text {hold }}=\frac{\hat{\mathbf{p}}^{2}}{2 m}+g\left|\tilde{\psi}_{a}(\mathbf{r}, t)\right|^{2}+g\left|\tilde{\psi}_{a}\left(v_{z} t \hat{e}_{z}-\mathbf{r}, t\right)\right|^{2} .
$$

From Eq. 25, we find

$$
\begin{array}{r}
\left|\tilde{\psi}_{a}\left(v_{z} t \hat{e}_{z}-\mathbf{r}, t\right)\right|^{2}= \\
\left|\tilde{\psi}_{a}(\mathbf{r}, t)\right|^{2}+\frac{2 \alpha_{z} v_{z} t z}{\lambda_{z}^{3}(t) \lambda_{\rho}^{2}(t)}-\frac{\alpha_{z} v_{z}^{2} t^{2}}{\lambda_{z}^{3}(t) \lambda_{\rho}^{2}(t)} .
\end{array}
$$

Plugging Eq. (32) into Eq. (31), we obtain

$$
\hat{\tilde{H}}_{\text {hold }}=\frac{\hat{\mathbf{p}}^{2}}{2 m}+2 g\left|\tilde{\psi}_{a}(\mathbf{r}, t)\right|^{2}-F_{z}(t) z+C(t) .
$$

Equation (33) implies that $C(t)$, which is independent of $\mathbf{r}$, contributes an overall phase to $\tilde{\psi}_{a}(\mathbf{r}, t)$ at each time $t$. The effective time-dependent force $F_{z}(t)$ along the negative $z$-direction,

$$
F_{z}(t)=-\frac{2 g \alpha_{z} v_{z} t}{\lambda_{z}^{3}(t) \lambda_{\rho}^{2}(t)}
$$

is due to the relative motion of the two components with respect to each other and the mean-field interactions.

We now make the assumption that the effective force term in Eq. (33) does not notably affect the time evolution of the density $\left|\tilde{\psi}_{a}(\mathbf{r}, t)\right|^{2}$. Under this assumption, the time evolution of the scaling factors $\lambda_{z}(t)$ and $\lambda_{\rho}(t)$ is governed by the differential equations derived by Castin and Dum from the scaling ansatz for a single-component BEC [14:

$$
\frac{d^{2} \lambda_{z}(t)}{d t^{2}}=\frac{\omega_{z}^{2}}{\lambda_{\rho}^{2}(t) \lambda_{z}^{2}(t)}
$$

and

$$
\frac{d^{2} \lambda_{\rho}(t)}{d t^{2}}=\frac{\omega_{\rho}^{2}}{\lambda_{\rho}^{3}(t) \lambda_{z}(t)}
$$

The black solid lines in Fig. 5(a) show $\lambda_{\rho}(t)$ and $\lambda_{z}(t)$, obtained by solving Eqs. (35)-(36) numerically for the same parameters as those employed in Fig. 4. Using these solutions, the solid line in Fig. 5(b) shows the effective force $F_{z}(t)$ as a function of the dimensionless time $t E_{R} / h$. The magnitude of the effective force first increases and then decreases with increasing time. As shown below, the turn-around time is, to leading order, given by the inverse of the transverse trapping frequency $\omega_{\rho}$.

While we assumed that the time dynamics of $\left|\tilde{\psi}_{a}(\mathbf{r}, t)\right|$ is largely independent of the effective force $F_{z}(t)$, we deduce from Sec. IIID that the time evolution of the phase $\tilde{\gamma}_{a}(\mathbf{r}, t)$ is non-negligibly impacted by $F_{z}(t)$. According to the momentum-impulse relationship, the impulse $I_{z}(t)$ imparted by the effective force on the system at time $t$ reads

$$
I_{z}(t)=\int_{0}^{t} F_{z}(\tau) d \tau
$$

The black solid line in Fig. 5(c) shows that the magnitude of $I_{z}(t)$ increases monotonically with increasing effective hold time. Using that the change of the momentum during the hold time is equal to $I_{z}(t)$, we estimate that the effective force $F_{z}(t)$ changes the phase $\tilde{\gamma}_{a}(\mathbf{r}, t)$ by $\phi_{\operatorname{lin}}(z, t)$,

$$
\phi_{\operatorname{lin}}(z, t)=\frac{I_{z}(t)}{\hbar} z,
$$

where $0^{+}<t<t_{\text {end }}$. We refer to this phase as "linear phase" since it depends linearly on $z$. It vanishes in the limit that the population of state $\tilde{\psi}_{b}(\mathbf{r}, t)$ does not move relative to that in state $\tilde{\psi}_{a}(\mathbf{r}, t)$.

The expansion of the two components for $0^{+}<t<$ $t_{\text {end }}$ introduces an additional contribution to the phase $\tilde{\gamma}_{a}(\mathbf{r}, t)$, which we refer to as quadratic phase $\phi_{\text {quad }}(z, \rho, t)$ due to its quadratic dependence on $z^{2}$ and $\rho^{2}$. The quadratic phase is independent of $v_{z}$ and analogous to the phase that develops during the expansion of a singlecomponent BEC [14,

$$
\phi_{\text {quad }}(z, \rho, t)=\frac{m z^{2}}{2 \hbar \lambda_{z}(t)} \frac{d \lambda_{z}(t)}{d t}+\frac{m \rho^{2}}{2 \hbar \lambda_{\rho}(t)} \frac{d \lambda_{\rho}(t)}{d t} .
$$

Combining the linear and quadratic phases, the expression for $\tilde{\gamma}_{a}(\mathbf{r}, t)$ reads

$$
\tilde{\gamma}_{a}(\mathbf{r}, t)=\phi_{\text {lin }}(z, t)+\phi_{\text {quad }}(z, \rho, t) .
$$

The second $\pi / 2$-pulse, applied at $t=t_{\text {end }}$, rotates the spinor at each $\mathbf{r}$ by $-\pi / 2$ about the $x$-axis on the Bloch sphere.

The division of the phase $\tilde{\gamma}_{a}(\mathbf{r}, t)$ into a linear and a quadratic contribution was, to the best of our knowledge, first discussed in Ref. [25]. For later work see Refs. [16, 26]. Reference 25] measured, employing a theory framework motivated by Ref. [14, the linear and quadratic phases of a ${ }^{23} \mathrm{Na} \mathrm{BEC}$ using matter-wave Bragg interferometry (see Ref. [27] for a related measurement of 
the linear phase). Even though the $\pi / 2$ Bragg pulses are notably shorter than our $\pi / 2$-pulses, the scenario considered in Secs. IVD and IVE namely, the Ramsey-type pulse sequence for the lattice coupling case, is closely related to Ref. 25].

To push the analytical treatment even further, we approximate $\lambda_{z}(t)$ and $\lambda_{\rho}(t)$ by [14]

$$
\lambda_{\rho}(t) \approx \sqrt{1+\omega_{\rho}^{2} t^{2}}
$$

and

$$
\begin{array}{r}
\lambda_{z}(t) \approx \\
1+\left(\frac{\omega_{z}}{\omega_{\rho}}\right)^{2}\left[\omega_{\rho} t \arctan \left(\omega_{\rho} t\right)-\ln \sqrt{1+\omega_{\rho}^{2} t^{2}}\right] .
\end{array}
$$

The red dashed and blue dash-dotted lines in Fig. 5(a) show $\lambda_{z}(t)$ and $\lambda_{\rho}(t)$ obtained using these analytical expressions. The agreement with the numerical solutions to Eqs. (35)-(36) [see the solid lines in Fig. 5(a)] is excellent. As a consequence, the effective force $F_{z}(t)$ and impulse $I_{z}(t)$, calculated using the approximate results for the scaling parameters, nearly coincide with the results that are obtained using the numerically determined scaling factors [compare the red dashed and black solid lines in Figs. 5(b) and 5(c)]. While Fig. 5 focuses on one specific parameter combination, similarly convincing agreement is found for other parameter combinations.

To understand the non-monotonic behavior of the effective force displayed in Fig. 5(b), we plug Eqs. (41) and (42) into Eq. (34). Taylor-expanding around small $\omega_{z} / \omega_{\rho}$ and neglecting terms of order $\left(\omega_{z} / \omega_{\rho}\right)^{2}$ and higher, we obtain

$$
F_{z}(t) \approx-\frac{2 g \alpha_{z} v_{z}}{\omega_{\rho}}\left(\frac{1}{\omega_{\rho} t}+\omega_{\rho} t\right)^{-1}
$$

Thus, for $\omega_{\rho} t \ll 1$ and $\omega_{\rho} t \gg 1, F_{z}(t)$ is proportional to $-\omega_{\rho} t$ and $-\left(\omega_{\rho} t\right)^{-1}$, respectively. Within the approximations made, $F_{z}(t)$ takes on a global minimum for $\omega_{\rho} t=1$. For comparison, the minimum in Fig. 5(b) occurs at $t E_{R} / h \approx 1.5$, which corresponds to $\omega_{\rho} t \approx 0.96$.

Equipped with fully analytical expressions for $\tilde{\psi}_{a}(\mathbf{r}, t)$ and $\tilde{\psi}_{b}(\mathbf{r}, t)$, we are ready to compare the spin dynamics obtained within this Thomas-Fermi approximation-like framework to the spin dynamics obtained by solving the Gross-Pitaevskii equation numerically. To this end, the third and fourth columns of Fig. 4 show the same observables as the first and second columns. While the first and second columns are obtained-as discussed in detail in Sec. IIID - by analyzing the solutions to the full Gross-Pitaevskii equation, the third and fourth columns are obtained using our fully analytical solutions derived above. A quick comparison indicates that the overall agreement is strikingly good. This a posteriori justifies the assumptions made in developing the analytical framework presented in this section. Most importantly, the good agreement allows us to unambiguously state that

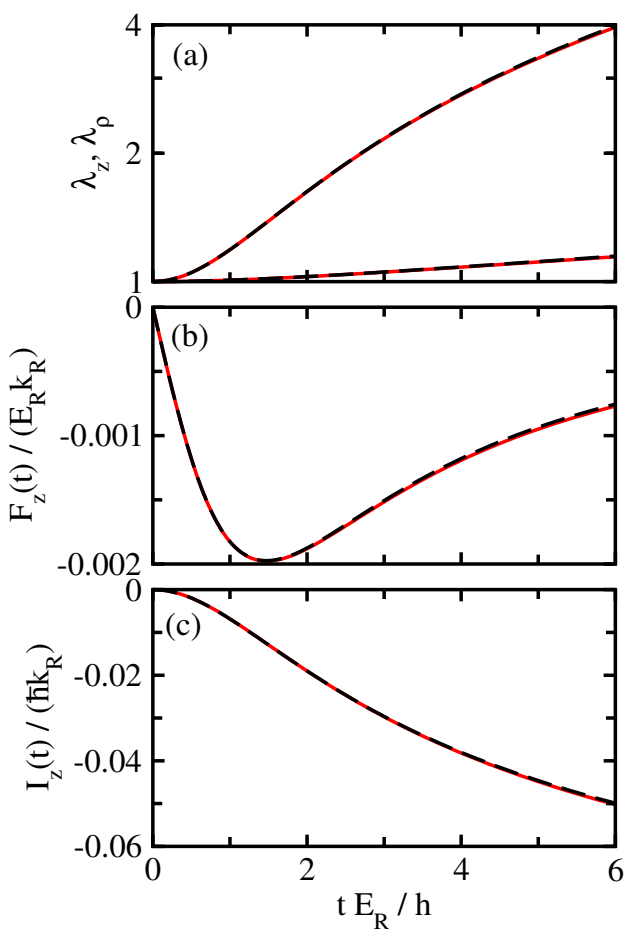

FIG. 5: Characteristics of the analytical framework discussed in Sec. IIIE Scaling parameters, effective force, and impulse during the hold time of the Raman-Ramsey-type pulse sequence for a ${ }^{87} \mathrm{Rb}$ BEC. Results are shown for $E_{R} / h=$ $1960 \mathrm{~Hz}, \Omega_{0, R}=E_{R}, \delta_{R}=0, N=3 \times 10^{5}, \omega_{\rho}=2 \pi \times 200 \mathrm{~Hz}$, and $\omega_{z}=2 \pi \times 40 \mathrm{~Hz}$. (a) The red dashed and blue dashdotted lines show the scaling parameters $\lambda_{z}(t)$ and $\lambda_{\rho}(t)$, respectively, obtained using the formula given in Eqs. (41) and 42 ; note that the vertical axis employs a logarithmic scale. (b) The red dashed line shows the effective force $F_{z}(t)$ calculated using our analytical expressions for the scaling parameters in Eq. (34). (c) The red dashed line shows the impulse $I_{z}(t)$ calculated using our analytical expressions for the scaling parameters in Eq. (37). For comparison, the solid lines in panels (a)-(c) show results obtained by numerically solving the differential equations for $\lambda_{\rho}(t)$ and $\lambda_{z}(t)$. The excellent agreement between the analytical and numerical results validates the use of the analytical expressions for the scaling parameters.

both the linear phase and the quadratic phase need to be accounted for to obtain a faithful description of the interference fringes.

Section IVD returns to the theoretical framework developed in this section. The analytical framework for the Raman Ramsey-type sequence, which relies heavily on the assumption that all four coupling strengths $g_{i j}$ are (approximately) equal to each other, cannot be applied directly to the lattice Ramsey-type sequence since the corresponding two-state model is described by coupling strengths $g_{a a}=g_{b b}=g_{a b} / 2=g_{b a} / 2$. Despite of this, it is argued in Sec. IVD that the model developed here provides important insights for the lattice coupling case as well. 


\section{LATTICE COUPLING CASE}

\section{A. Two-state model}

For the lattice case, all atoms occupy the same hyperfine state; our calculations reported below are for ${ }^{87} \mathrm{Rb}$ atoms in the $\left|F, m_{F}\right\rangle=|1,-1\rangle$ state. Assuming a single mean-field wave function $\Phi(\mathbf{r}, t)$, the Hamiltonian $\hat{H}$ to be used in the time-dependent Gross-Pitaevskii equation reads 28

$$
\hat{H}=\frac{\hat{\mathbf{p}}^{2}}{2 m}+V_{\text {trap }}(\mathbf{r}, t)+V_{\text {lat }}(\mathbf{r}, t)+g_{a a}|\Phi(\mathbf{r}, t)|^{2},
$$

where the one-dimensional moving lattice potential $V_{\text {lat }}(\mathbf{r}, t)$ is given by

$$
V_{\text {lat }}(\mathbf{r}, t)=2 \Omega_{L}(t) \cos ^{2}\left(k_{L} z-\frac{\omega_{L}}{2} t\right)
$$

and $\Phi(\mathbf{r}, t)$ is normalized according to $\int|\Phi(\mathbf{r}, t)|^{2} d \mathbf{r}=$ 1. Figure 6(a) shows the density $|\Phi(\mathbf{r}, t)|^{2}$, obtained by solving the time-dependent mean-field equation for the Hamiltonian given in Eq. 44 for typical experimental parameters, as a function of $z$ for $\rho=0$ and a time corresponding to a $\pi / 2$-pulse, i.e., for $t=\pi h /\left(2 \Omega_{0, L}\right)$. In this example, the BEC is prepared in the ground state of the harmonic trap. At time $t=0$, the trapping potential is turned off and the lattice with $E_{L} / h=1960 \mathrm{~Hz}, \omega_{L}=$ $4 E_{L} / \hbar$ and $\Omega_{0, L}=E_{L}$ is flashed on for $0.1276 \mathrm{~ms}$. Here, the coupling strength $\Omega_{0, L}$ is chosen to be comparable to the chemical potential $\mu$ of the BEC at $t=0\left(\Omega_{0, L} \approx\right.$ $0.59 \mu)$. The size of the BEC does not change notably during the duration of the lattice pulse: it extends over approximately 80 lattice sites. Figure 6(a) shows that the lattice pulse "imprints" fine oscillations along the $z$ direction onto the mean-field density.

To facilitate the analysis, it is desirable to bring out the intrinsic dynamics by rotating the lattice induced oscillations away. As we discuss in the next paragraphs, this can be accomplished within the framework of an approximate two-state model, which assumes that the BEC only occupies momenta along the $z$-direction near $\hbar k_{z}=0$ and $\hbar k_{z}=2 \hbar k_{L}$ and not near $n \hbar k_{L}$ with $n=-2, \pm 4, \pm 6, \cdots$. This assumption is well justified for the example shown in Fig. 6(a). The density cut in momentum space [Fig. 6(b)] shows peaks centered near $\hbar k_{z}=0$ and $\hbar k_{z}=2 \hbar k_{L}$; the populations of these peaks are $65.46 \%$ and $33.79 \%$, respectively. Since the peaks centered near $\hbar k_{z}=-2 \hbar k_{L}$ and $\hbar k_{z}=4 \hbar k_{L}$ have tiny populations $(0.665 \%$ and $0.084 \%$, respectively), the two-state model developed below is expected to capture the dynamics of this system semi-quantitatively. More generally, the applicability of the two-state model requires that the lattice pulse or pulses are sufficiently short and sufficiently weak. The two-state model introduced below can be improved systematically by accounting for successively more "momentum components", i.e., by increasing the number of $n$ values included in Eq. 46. In the limit of an infinite
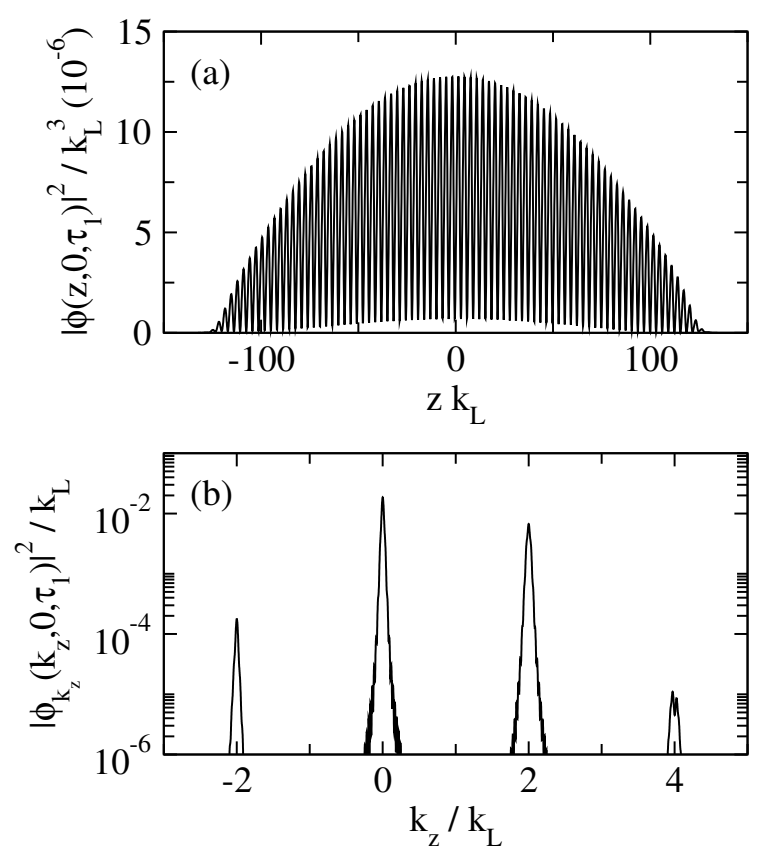

FIG. $6:{ }^{87} \mathrm{Rb}$ BEC density after the application of a $\pi / 2$ lattice pulse (numerical results). The solid lines in (a) and (b) show density cuts as a function of $z k_{L}$ (real space) and $k_{z} / k_{L}$ (momentum space), respectively, for $t_{\text {start }}=0, N=3 \times 10^{5}$, $E_{L} / h=1960 \mathrm{~Hz}$ (corresponding to $k_{L}=5.81 \mu \mathrm{m}^{-1}$ ), $\Omega_{0, L}=E_{L}, \delta_{L}=0, \omega_{\rho}=2 \pi \times 200 \mathrm{~Hz}$, and $\omega_{z}=2 \pi \times 40 \mathrm{~Hz}$. The real space density cut $\left|\Phi\left(z, 0, \tau_{1}\right)\right|^{2}$ is governed by fine oscillations that are related to the fact that the BEC contains, after the application of the lattice pulse, non-zero momentum components. The momentum space cut $\left[\left|\Phi_{k_{z}}\left(k_{z}, 0, \tau_{1}\right)\right|^{2}\right.$ is obtained by taking the square of the Fourier transform of $\left.\Phi\left(z, 0, \tau_{1}\right)\right]$ shows that the BEC density is governed by momenta centered around $\hbar k_{z} \approx 0$ and $\hbar k_{z} \approx 2 \hbar k_{L}$.

state model that accounts for all $n(n=0, \pm 2, \cdots)$ the description is equivalent to that captured by the original mean-field Hamiltonian [Eq. (44) with $V_{\text {lat }}(\mathbf{r}, t)$ given by Eq. (45)]. 33

To derive the two-state model, we make the ansatz 32 ,

$$
\Phi(\mathbf{r}, t)=\tilde{\psi}_{a}(\mathbf{r}, t)+\tilde{\psi}_{b}(\mathbf{r}, t) \exp \left(2 \imath k_{L} z\right)
$$

where $\tilde{\psi}_{a}(\mathbf{r}, t)$ and $\tilde{\psi}_{b}(\mathbf{r}, t)$ are assumed to be localized in the vicinity of the momenta $\hbar k_{z}=0$ and $\hbar k_{z}=2 \hbar k_{L}$, respectively. The functions $\tilde{\psi}_{a}(\mathbf{r}, t)$ and $\tilde{\psi}_{b}(\mathbf{r}, t)$ are normalized according to Eq. (8) and Eq. (9) with $\psi_{j}(\mathbf{r}, t)$ replaced by $\tilde{\psi}_{j}(\mathbf{r}, t)$. Since the widths of the momentum distributions associated with the states $\tilde{\psi}_{a}(\mathbf{r}, t)$ and $\tilde{\psi}_{b}(\mathbf{r}, t)$ are assumed to be narrow compared to $2 \hbar k_{L}$ [this is, indeed, the case for the example shown in Fig. 6(b)], we demand that the "separation condition"

$$
\int \exp \left(\imath 2 k_{L} z\right) \tilde{\psi}_{a}(\mathbf{r}, t)\left[\tilde{\psi}_{b}(\mathbf{r}, t)\right]^{*} d \mathbf{r}=0
$$


holds.

Following the standard mean-field approach, we write the $N$-body wave function as a product over singleparticle orbitals, namely as $\Phi\left(\mathbf{r}_{1}, t\right) \Phi\left(\mathbf{r}_{2}, t\right) \cdots \Phi\left(\mathbf{r}_{N}, t\right)$. Variation of the energy functional with respect to $\left[\tilde{\psi}_{a}(\mathbf{r}, t)\right]^{*}$ and $\left[\tilde{\psi}_{b}(\mathbf{r}, t)\right]^{*}$ then yields two coupled nonlinear equations, namely Eq. 12 with $\hat{H}$ replaced by $\hat{\tilde{H}}_{2 \text {-st }}$, where

$$
\begin{aligned}
\hat{\tilde{H}}_{2-\text { st }}= & \left(\begin{array}{cc}
\hat{\mathbf{p}}^{2} \\
2 m
\end{array}+V_{\text {trap }}(\mathbf{r}, t)\right) \otimes I_{2}+\left(\begin{array}{cc}
g\left|\tilde{\psi}_{a}(\mathbf{r}, t)\right|^{2}+2 g\left|\tilde{\psi}_{b}(\mathbf{r}, t)\right|^{2} & 0 \\
0 & 2 g\left|\tilde{\psi}_{a}(\mathbf{r}, t)\right|^{2}+g\left|\tilde{\psi}_{b}(\mathbf{r}, t)\right|^{2}
\end{array}\right)+ \\
& \left(\begin{array}{cc}
0 & \frac{\Omega_{L}(t)}{2} \\
\frac{\Omega_{L}(t)}{2} & \frac{2 \hbar k_{L} \hat{p}_{z}}{m}+\delta_{L}
\end{array}\right) .
\end{aligned}
$$

In deriving Eq. 48, we assumed that integrals such as

$$
\int\left|\tilde{\psi}_{j}(\mathbf{r}, t)\right|^{2} \exp \left( \pm n \imath k_{L} z\right) d \mathbf{r}
$$

( $n=2,4, \cdots)$, which have rapidly oscillating integrands, vanish. This means that portions of the kinetic energy, lattice potential, trap potential, and mean-field energy contributions are neglected in Eq. (48).

Comparison of the approximate two-state lattice Hamiltonian [Eq. 480] and the rotated Raman Hamiltonian [Eq. (14)] shows that the two Hamiltonians agree if we enforce that $E_{R}, \Omega_{R}(t)$, and $\delta_{R}$ are equal to $E_{L}$, $\Omega_{L}(t)$, and $\delta_{L}$, respectively and if additionally the following holds: $g_{a a}=g_{b b}=g$ and $g_{a b}=2 g$. For the $F=1$ hyperfine manifold of ${ }^{87} \mathrm{Rb}$, the mean-field interactions of the two Hamiltonians do not agree since we have $g_{a a} \approx g_{b b} \approx g_{a b}$. Consequently, the dynamics for the Raman coupled and lattice coupled systems are expected to differ even if the single-particle coupling mechanisms are characterized by matching parameters. In what follows, we will focus on the interaction-induced differences.

Since the population in state $\tilde{\psi}_{a}(\mathbf{r}, t)\left[\tilde{\psi}_{b}(\mathbf{r}, t)\right]$ experiences a mean-field interaction due to the population in state $\tilde{\psi}_{b}(\mathbf{r}, t)\left[\tilde{\psi}_{a}(\mathbf{r}, t)\right]$ that is about two times larger for the lattice coupled Hamiltonian than for the Raman coupled Hamiltonian, the lattice coupled system has a stronger tendency to phase separate than the Raman coupled system (this argument uses the fact that $g$ is positive for the $F=1$ hyperfine manifold of ${ }^{87} \mathrm{Rb}$ ). Phase separation has been discussed in the literature in the context of multi-component BECs 29. The framework developed here may also provide an intuitive understanding of the formation of the ferromagnetic domains observed in Ref. 30.

The difference between the Raman and lattice coupling cases can also be interpreted from an alternative viewpoint. To this end, we rewrite the mean-field terms from Eq. 48 as

$$
\begin{array}{r}
g\left|\tilde{\psi}_{a}(\mathbf{r}, t)\right|^{2}+2 g\left|\tilde{\psi}_{b}(\mathbf{r}, t)\right|^{2}= \\
g_{\mathrm{eff}}\left[\left|\tilde{\psi}_{a}(\mathbf{r}, t)\right|^{2}+\left|\tilde{\psi}_{b}(\mathbf{r}, t)\right|^{2}\right]-g\left|\tilde{\psi}_{a}(\mathbf{r}, t)\right|^{2}
\end{array}
$$

and

$$
\begin{array}{r}
g\left|\tilde{\psi}_{b}(\mathbf{r}, t)\right|^{2}+2 g\left|\tilde{\psi}_{a}(\mathbf{r}, t)\right|^{2}= \\
g_{\mathrm{eff}}\left[\left|\tilde{\psi}_{a}(\mathbf{r}, t)\right|^{2}+\left|\tilde{\psi}_{b}(\mathbf{r}, t)\right|^{2}\right]-g\left|\tilde{\psi}_{b}(\mathbf{r}, t)\right|^{2}
\end{array}
$$

where $g_{\text {eff }}$ is defined to be equal to $2 g$. The right hand sides of Eqs. (50) and (51) suggest that the difference between the lattice and Raman coupling cases is due to two things: First, $g_{a a}, g_{b b}$, and $g_{a b}$ can be identified to be equal to $g_{\text {eff }}$, suggesting that the lattice coupled system is characterized by a two times stronger repulsion than the Raman coupled system. Second, there exists an effective on-site attraction in the two-state model of the lattice coupled system of strength $-g$ [31] that has no analog in the Raman coupled system.

We emphasize that the two interpretations introduced above are consistent with the scattering diagram arguments outlined in Sec. I. The "factor of 2 " in the second $2 \times 2$ matrix on the right hand side of Eq. 48 is due to non-vanishing scattering matrix elements; the analogous scattering matrix elements vanish in the Raman coupled case due to the orthogonality of the two different hyperfine states.

\section{B. Rabi oscillations: Theory overview}

This section discusses lattice coupling induced Rabi oscillations. Figure 7 compares the mean-field results for the full lattice Hamiltonian [Eqs. 44) and 45); solid black lines] with those obtained using the approximate two- and four-state Hamiltonians (red dashed and blue dotted lines, respectively). For all 9 parameter combinations considered in Fig. 7] the four-state model reproduces the dynamics obtained for the full mean-field lattice Hamiltonian extremely well. While the two-state model results deviate somewhat from the results for the full lattice Hamiltonian, the two-state model captures the main features of the Rabi oscillations such as the change of the damping of the Rabi oscillations with increasing number of particles [Figs. 7)(ai)-(aiii)] and with increas- 

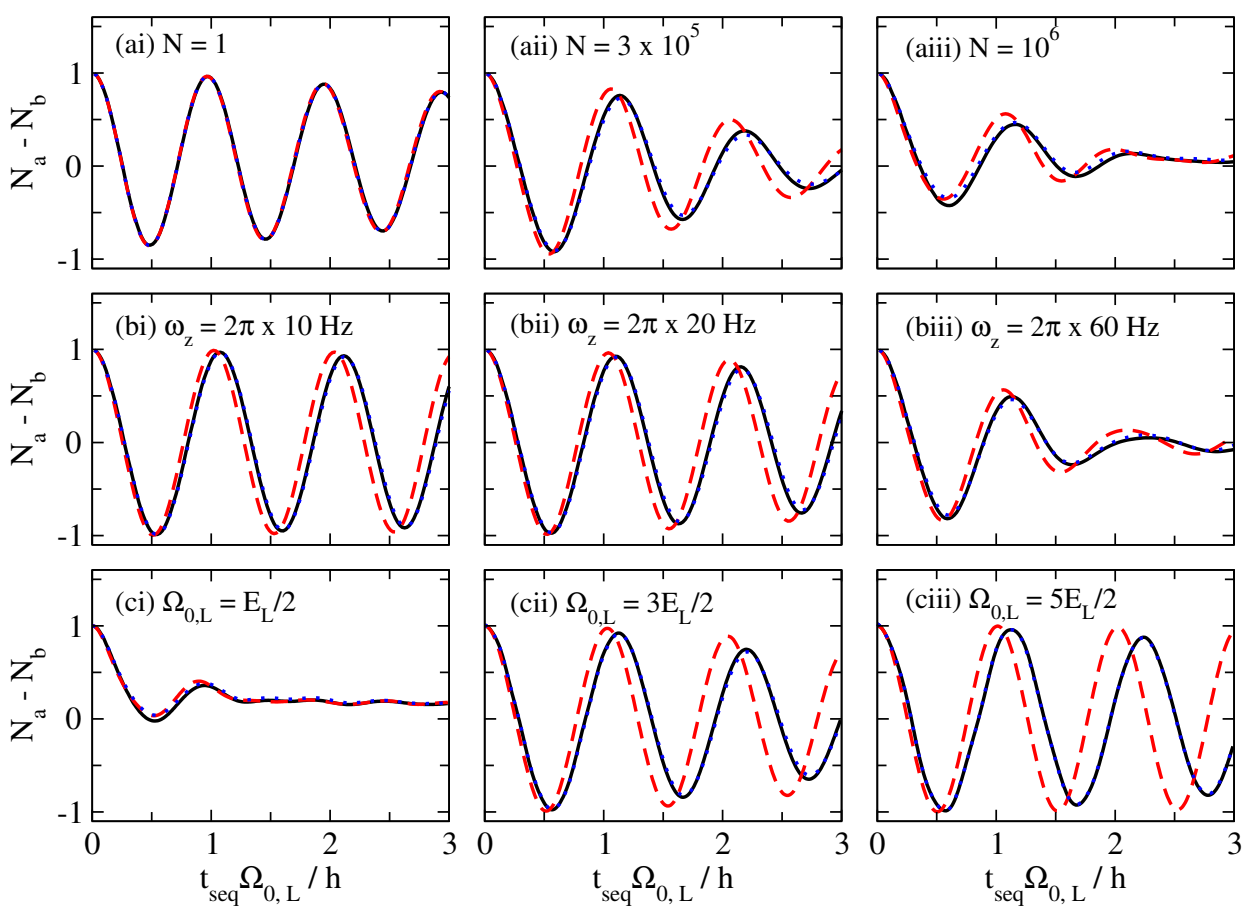

FIG. 7: Rabi oscillations for lattice coupling case (numerical results). The lines show the difference $N_{a}-N_{b}$ between the fractional populations as a function of the dimensionless time $t_{\text {seq }} \Omega_{0, L} / h$ for $t_{\text {start }}=0, E_{L} / h=1960 \mathrm{~Hz}, \delta_{L}=0$, and $\omega_{\rho}=2 \pi \times 200 \mathrm{~Hz}$. The black solid, red dashed, and blue dotted lines show results obtained by solving the time-dependent mean-field equation for the full lattice Hamiltonian [Eqs. (44) and [45], the approximate two-state Hamiltonian [Eq. 448], and the approximate four-state Hamiltonian (this Hamiltonian is not written out explicitly in the text). The black solid and blue dotted lines nearly coincide (in particular, the blue dotted lines are hardly visible on the scale shown). (ai)-(aiii) Changing the particle number $N$ (the values are given in the panels). The weak angular trapping frequency is $\omega_{z}=2 \pi \times 40 \mathrm{~Hz}$ and the coupling strength is $\Omega_{0, L}=E_{L}$. (bi)-(biii) Changing the angular trapping frequency $\omega_{z}$ (the values are given in the panels). The coupling strength is $\Omega_{0, L}=E_{L}$ and the number of particles is $N=3 \times 10^{5}$. (ci)-(ciii) Changing the coupling strength $\Omega_{0, L}$ (the values are given in the panels). The number of particles is $N=3 \times 10^{5}$ and the weak trapping frequency is $\omega_{z}=2 \pi \times 40 \mathrm{~Hz}$.

ing strength of the weak trapping frequency [Figs. 7(bi)(biii)]. Moreover, the rapid reduction of the oscillation amplitude for small coupling strength [Fig. 7(ci) is for $\left.\Omega_{0, L}=E_{L} / 2\right]$ is remarkably well captured by the approximate two-state model. For larger lattice strengths [Figs. 7. (bii) and (biii) are for $\Omega_{0, L}=3 E_{L} / 2$ and $5 E_{L} / 2$,

Since the approximate two-state model provides a qualitatively and for some parameter combinations even a (semi-)quantitatively correct description of the dynamics, it is instructive to compare the Rabi oscillations for the lattice and Raman coupled systems. If the two-state lattice model is exact, the difference between the Rabi oscillations for the lattice and Raman coupled systems will be - assuming that the small differences between $g_{a a}, g_{b b}$, and $g_{a b}$ do not play a role - solely due to the "factor of 2 " discussed in Sec. IVA. The parameters in Figs. 2 and 7 are chosen such that the solid line in Fig. 2(a) can be directly compared with the curves in Fig. 7(ai), the dashed line in Fig. 2(a) with the curves in Fig. 7)(aii), and the respectively], in contrast, the two-state model captures the period of the Rabi oscillations comparatively poorly. The reason is that larger lattice coupling strengths lead to enhanced and non-negligible occupations of momenta centered near $\hbar k_{z} \approx-2 \hbar k_{L}$ and $\hbar k_{z} \approx 4 \hbar k_{L}$.

dotted line in Fig. 2(a) with the curves in Fig. 7(aiii). An analogous correspondence exists for Fig. 2(b) and Figs. 7(bi)-(biii) as well as for Fig. 2(c) and Figs. 7(ci)(ciii). A careful comparison of Figs. 7 and 2 indicates that the most prominent effect of the factor of 2 is to significantly enhance the damping or dephasing of the Rabi oscillations.

In what follows we attempt to pinpoint why the factor of 2 (lattice coupling case) enhances the damping compared to the case where this factor is equal to 1 (Raman coupling case). To start this discussion, we remind the reader that the analytical treatment in Sec. IIIE, which assumes vanishing detuning, relies heavily on the 
assumption that there exists a symmetry between the components $\tilde{\psi}_{a}(\mathbf{r}, t)$ and $\tilde{\psi}_{b}(\mathbf{r}, t)$ [see Eq. 29p]. In fact, one can show that this symmetry is - within the Thomas-Fermi approximation - an exact symmetry provided $g_{a a}=g_{b b}=g_{a b}$. Intuitively, this symmetry can be understood by realizing that the strength of the scattering between two atoms in the same hyperfine state is identical to that of the scattering between two atoms in different hyperfine states. This implies that neither the two-body interactions nor the Raman coupling (recall, we are considering the zero detuning scenario) bias populations to one hyperfine state over another. In the lattice coupling case with $\delta_{L}=0$, the factor of 2 breaks the symmetry. The effective attractive on-site interactions [see the discussion in the context of Eqs. (50) and (51)], which can alternatively be interpreted as effective repulsive off-site interactions, favor configurations that reduce the overlap between the densities $\left|\tilde{\psi}_{a}(\mathbf{r}, t)\right|^{2}$ and $\left|\tilde{\psi}_{b}(\mathbf{r}, t)\right|^{2}$. Since the effective repulsive off-site interactions depend on the density, they vary spatially. This spatial dependence can result in a shape of the density $\left|\tilde{\psi}_{a}(\mathbf{r}, t)\right|^{2}$ that is different from that of the density $\left|\tilde{\psi}_{b}(\mathbf{r}, t)\right|^{2}$. If this occurs, the fractional population difference varies locally, leading to a spatially dependent population transfer and, correspondingly, a damping or dephasing of the Rabi oscillations. In a complementary picture, the effective repulsive off-site interactions can be thought of as an effective spatially and temporally varying coupling term. In this picture, the damping of the Rabi oscillations emerges naturally. Section IV D makes this discussion concrete for a $\pi / 2$ pulse (first step of the Ramsey-type sequence).

\section{Rabi oscillations: Theory-experiment comparison}

The symbols in Figs. 8(a) and 8(b) show experimental data for Rabi oscillations induced by a moving optical lattice with weak and strong coupling, respectively. The excellent agreement between the solutions to the Gross-Pitaevskii equations for the full lattice Hamiltonian (solid lines) and the experimental data indicates that the experiments operate in the mean-field regime, i.e., the Gross-Pitaevskii framework captures the population transfer between the two momentum components quantitatively. The approximate two-state model (dotted lines) provides, as already discussed in the previous section, a semi-quantitative description of the latticeinduced Rabi oscillations in the weak coupling regime [Fig. 8(b)]; as such, it provides a meaningful conceptual framework for interpreting the results and contrasting the lattice- and Raman-induced Rabi oscillations.

A fit of the Rabi oscillation data obtained by solving the Gross-Pitaevskii equation for the full lattice Hamiltonian to Eq. (16) yields coupling strengths that are, respectively, $5 \%$ and $6 \%$ lower than those used in the simulations. This shows that the interactions do impact the
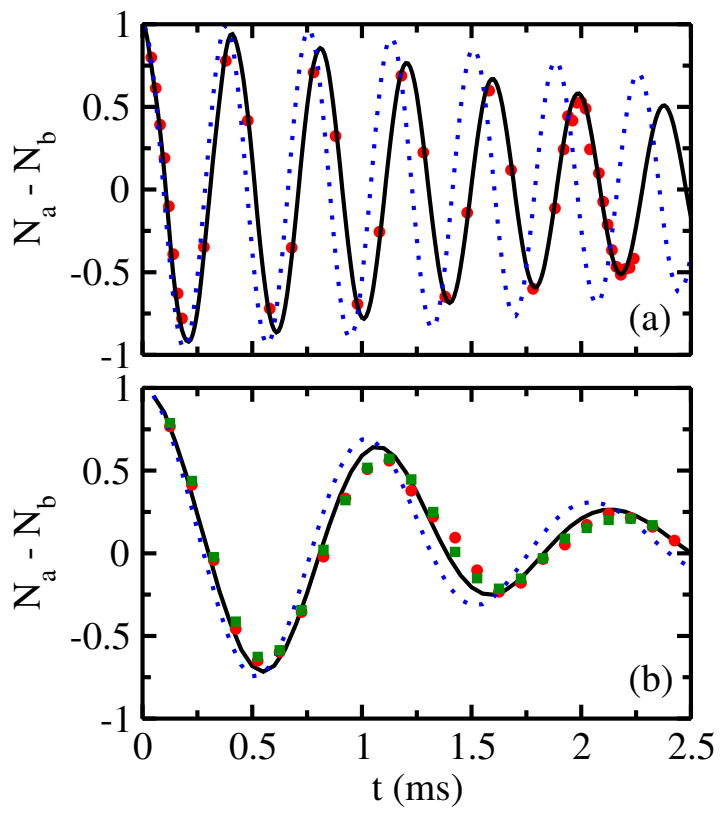

FIG. 8: Theory-experiment comparison for lattice Rabi oscillations with "strong" and "weak" coupling strengths for a ${ }^{87} \mathrm{Rb}$ BEC. The symbols show experimental data and the black solid lines show results from the Gross-Pitaevskii simulations for the full lattice Hamiltonian. For comparison, the blue dotted lines show results obtained for the approximate two-state model. The experimental parameters common to both panels are $E_{L} / h=1080 \mathrm{~Hz}$ and $t_{\text {start }}=0.5 \mathrm{~ms}$. (a) "Strong coupling" $\left(\Omega_{0, L} / h=2646 \mathrm{~Hz}\right)$ : The experimentally measured parameters are $\omega_{x}=2 \pi \times 172 \mathrm{~Hz}, \omega_{y}=2 \pi \times 139 \mathrm{~Hz}$, $\omega_{z}=2 \pi \times 33.6 \mathrm{~Hz}, \delta_{L} / h=-264 \mathrm{~Hz}$, and $N=1.1 \times 10^{5}$. The calculations set $\omega_{\rho}$ equal to the mean of $\omega_{x}$ and $\omega_{y}$; all other parameters are taken from the experiment. The chemical potential $\mu$ prior to turning off the trap is $1.439 \mathrm{E}_{L}$. The meanfield energy per particle prior to turning off the trap and after the $0.5 \mathrm{~ms}$ expansion is $0.8881 E_{L}$ and $0.7149 E_{L}$, respectively. The red circles show the result from one experimental run. (b) "Weak coupling" $\left(\Omega_{0, L} / h=980 \mathrm{~Hz}\right)$ : The experimentally measured parameters are $\omega_{\rho}=2 \pi \times 146 \mathrm{~Hz}, \omega_{z}=2 \pi \times 28 \mathrm{~Hz}$, $\delta_{L}=0$, and $N=2.7 \times 10^{5}$. The transverse trap frequency is determined by performing measurements along one axis. The calculations use the parameters from the experiment. The chemical potential $\mu$ prior to turning off the trap is $1.830 E_{L}$. The mean-field energy per particle prior to turning off the trap and after the $0.5 \mathrm{~ms}$ expansion is $1.129 E_{L}$ and $0.9297 E_{L}$, respectively. The red circles and green squares show the results from two separate experimental runs.

Rabi oscillations and that calibration of the experimental lattice strength needs to proceed with care. We note that the fit to the data in Fig. 8(a) has a significantly lower $\chi^{2}$ than the fit to the data in Fig. 8(b). For the experimental data shown in Fig. 8, the coupling strength is calibrated by inducing Rabi oscillations of a very dilute ${ }^{87} \mathrm{Rb} \mathrm{BEC}$ for a relative large lattice coupling strength; in this case, the Rabi oscillation data display essentially no damping. This calibration run yields a power-to-coupling-strength conversion. Assuming that the coupling strength scales 
as the square root of the power, the calibration curve can be used in subsequent science runs that operate at other powers. The outlined approach assumes that the power fluctuations are negligible over the course of several hours; we have checked that this is the case in our setup.

\section{Ramsey-type pulse sequence: Numerical results}

This section discusses numerical results for the Ramsey-type pulse sequence with lattice coupling (vanishing detuning, i.e., $\left.\delta_{L}=0\right)$. Figure 9 shows lattice coupling results for the same parameters as used in Fig. 4 (recall, Fig. 4 shows results for the Ramsey-type sequence

After the first $\pi / 2$-pulse [Figs. 9(ai) and 9(avi)], the population is distributed unequally among the two components, i.e., component $\tilde{\psi}_{a}(\mathbf{r}, t)$ has a larger population than component $\tilde{\psi}_{b}(\mathbf{r}, t)$; a 50/50 mixture is realized for a pulse of length $0.2958 \mathrm{~ms}$, i.e., for a pulse that is 2.3 times longer than the $\pi / 2$-pulse employed in Fig. 9 . Near the edge of the cloud, the density cuts $\left|\tilde{\psi}_{a}\left(z, 0, \tau_{1}\right)\right|^{2}$ and $\left|\tilde{\psi}_{b}\left(z, 0, \tau_{1}\right)\right|^{2}$ coincide to a good approximation. In the central region, in contrast, they differ. While the first component profile, $\left|\tilde{\psi}_{a}\left(z, 0, \tau_{1}\right)\right|^{2}$, approximately follows a Thomas-Fermi profile, the second component profile, $\left|\tilde{\psi}_{b}\left(z, 0, \tau_{1}\right)\right|^{2}$, is flatter than a Thomas-Fermi profile. Correspondingly, the local spin expectation value $\sigma_{z}\left(z, 0, \tau_{1}\right)$ has a roughly Gaussian shape as opposed to following a linear curve as in the Raman coupled case. This indicates, in agreement with the more general discussion at the end of Sec. IVB, that population from the center of the cloud is pushed toward the edge of the cloud due to the larger local effective repulsive off-site interaction at the center of the cloud compared to the edge. Using the Bloch-sphere picture, the spatially and temporally dependent effective repulsive off-site interaction or coupling leads to a spatially and temporally dependent torque along the $x$-direction during the first $\pi / 2$ pulse. This is confirmed by the local spin expectation value $\sigma_{y}\left(z, 0, \tau_{1}\right)$, whose spatial dependence differs from that of the densities of the components.

Altogether, the discussion shows that the interactions can, for the relatively weak lattice coupling strength of $\Omega_{0, L}=E_{L}$ considered in Fig. 9, not be neglected during the first $\pi / 2$-pulse, i.e., the lattice coupling is not sufficiently strong to prevent the system from rearranging structurally. As a consequence, the Rabi oscillations are damped or dephase. For the parameters chosen in Fig. 9, the factor of 2 leads to a notable cloud deformation during the first $\pi / 2$ pulse. For other parameter combinations, the densities of the components may deform more slowly, thereby leading to a slower damping or dephasing of the Rabi oscillations. with Raman coupling). The first and second columns in Fig. 9 are obtained by solving the time-dependent meanfield equation for the full lattice Hamiltonian while the third and fourth columns are obtained by solving the time-dependent mean-field equation for the approximate two-state lattice model. It can be seen that the results for the approximate two-state lattice Hamiltonian agree with those for the full lattice Hamiltonian rather well. Since the approximate two-state lattice model describes the dynamics faithfully, we use it below to gain insights into the results after the first $\pi / 2$-pulse [Figs. 9 (bi) and 9(bvi)], after the hold time [Figs. 9 (bii), 9 (bvii), 9 (biii), and 9 (bviii)], and after the second $\pi / 2$-pulse [Figs. 9 (biv), 9 (bix), 9(bv), and 9(bx)].

During the hold time, the amplitude and phase evolution is, as in the Raman coupled case, governed by the interplay between the interactions and the expansion. Because of the deviation of the component densities from the Thomas-Fermi profile and the "absence of symmetry" (see the discussion above), we were not able to develop an analytical framework that describes the dynamics during the hold time. However, comparing the second and third rows of Fig. 9 with the second and third rows of Fig. 4 , rough similarities between the time dynamics during the hold time for the two distinct coupling mechanisms can be recognized. Thus, while we do not have an analytical description, the formulation developed in the context of the Raman coupling case can serve as a crude zerothorder guide.

In what follows, we point out three aspects that are distinct for the lattice coupling case: (i) The local spin expectation value $\sigma_{y}\left(z, 0, \tau_{1}\right)$ is not symmetric with respect to $z=0$; this asymmetry persists during the hold time. (ii) During the hold time, the shapes of the densities $\left|\tilde{\psi}_{a}(z, 0, t)\right|^{2}$ and $\left|\tilde{\psi}_{b}(z, 0, t)\right|^{2}$ continue to change appreciably. (iii) The densities of the components and the local spin expectation value $\sigma_{y}(z, 0, t)$ develop spatial modulations during the hold time in the region where the two components are not spatially overlapping. These spatial modulations are more pronounced than in the Raman coupling case.

The second $\pi / 2$-pulse "transfers" the information encoded in $\sigma_{y}(\mathbf{r}, t)$ to the population difference $\sigma_{z}(\mathbf{r}, t)$. Since the cloud expands a fair bit during the hold time, the interactions can, to a good approximation, be neglected during the second $\pi / 2$-pulse. Consequently, the resulting densities of the components display a fringe or interference pattern. However, unlike in the Raman coupled case, the densities in the lattice coupled case are highly non-symmetric. As argued above, this asymmetry can be interpreted as a fingerprint of the fact that one of the terms on the diagonals in the second $2 \times 2$ matrix on the right hand side of Eq. 48 is multiplied by 


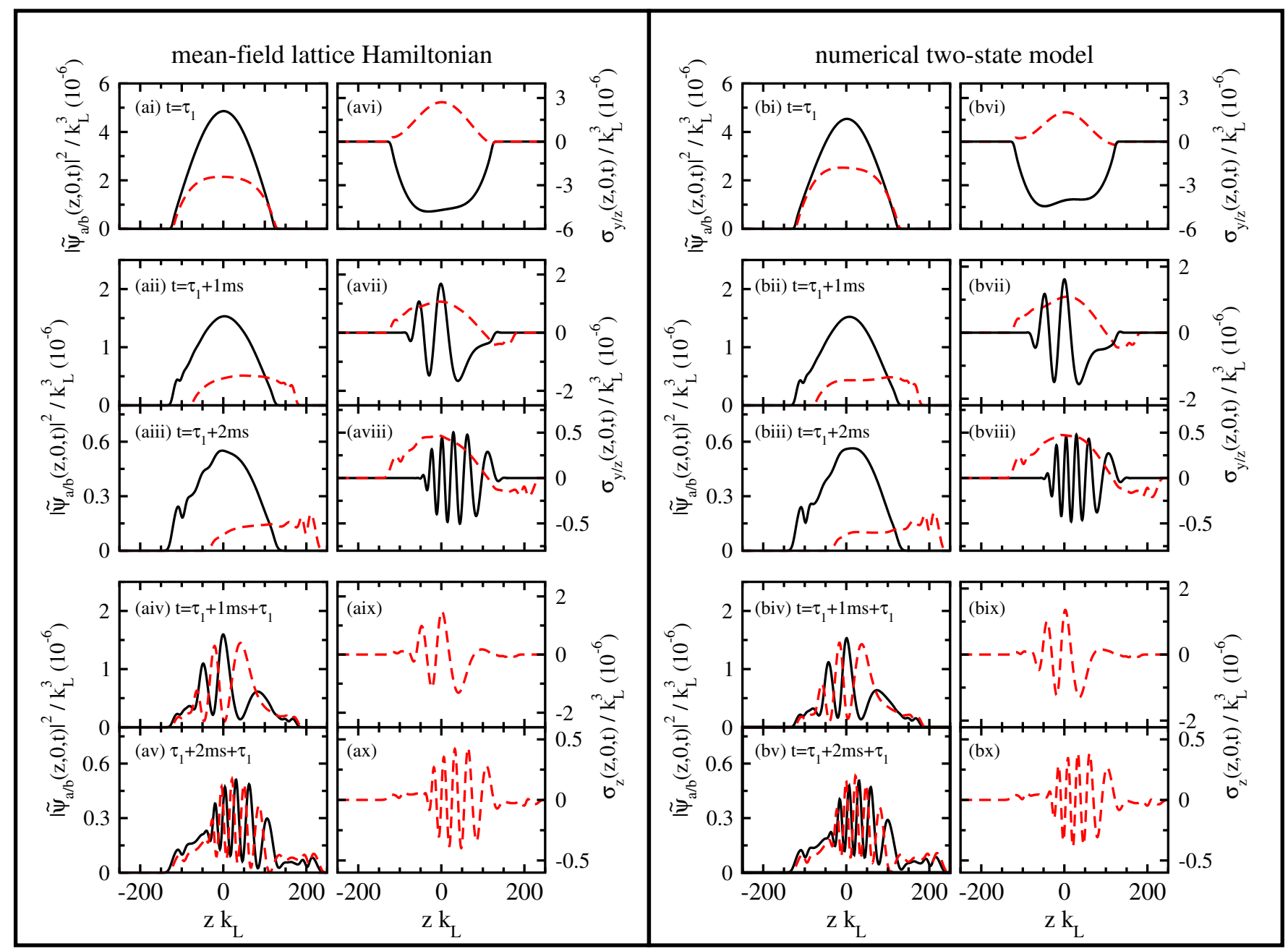

FIG. 9: Density cuts and local spin expectation values for the Ramsey-type pulse sequence with $E_{L} / h=1960 \mathrm{~Hz}, \Omega_{0, L}=E_{L}$, and $\delta_{L}=0$ (numerical results); these are the same parameters as used in Figs. 6 and 7(aii). The ${ }^{87} \mathrm{Rb}$ BEC consists of $N=3 \times 10^{5}$ atoms and is prepared in an axially symmetric trap with $\omega_{\rho}=2 \pi \times 200 \mathrm{~Hz}$ and $\omega_{z}=2 \pi \times 40 \mathrm{~Hz}$ (these are the same parameters as those used in Fig. 4). All results are obtained for $t_{\text {start }}=0$. The first and second columns are obtained by solving the time-dependent mean-field equation for the full lattice Hamiltonian [Eq. 444 with $V_{\text {lat }}(\mathbf{r}, t)$ given by Eq. (45)] numerically. The third and fourth columns show the same observables as the first and second columns but are, instead, obtained using the approximate two-state model introduced in Sec. IVA the agreement is quite good. The black solid and red dashed lines in panels (ai)-(av) show the density profiles $\left|\tilde{\psi}_{a}(z, 0, t)\right|^{2}$ and $\left|\tilde{\psi}_{b}(z, 0, t)\right|^{2}$, respectively. The black solid and red dashed lines in panels (avi)-(ax) show the local spin expectation values $\sigma_{y}(z, 0, t)$ and $\sigma_{z}(z, 0, t)$, respectively. The time increases from the first row, to the second/third row, to the fourth/fifth row (the value of the time is given in the panels); the second and fourth row correspond to a hold time of $1 \mathrm{~ms}$, and the third and fifth row correspond to a hold time of $2 \mathrm{~ms}$. Unlike in Fig. 4 . the first $\pi / 2$-pulse does not lead to $50 / 50$ mixture. This population imbalance after the first $\pi / 2$-pulse contributes to the development of unequally spaced interference fringes.

a factor of 2 .

\section{E. Ramsey-type pulse sequence: Theory-experiment comparison}

Figures 10(a) and 10(b) compare experimentally determined integrated densities (red circles) and theoretical results (black solid lines) for the Ramsey-type pulse sequence with lattice coupling for hold times of $0.5 \mathrm{~ms}$ and $1 \mathrm{~ms}$, respectively. The black solid lines are obtained by solving the Gross-Pitaevskii equation for the full lattice Hamiltonian and explicitly simulating the $12 \mathrm{~ms}$ of timeof-flight expansion after the Ramsey-type pulse sequence. For comparison, the blue dotted and green dashed lines show the results from the two-state model; the agreement with the full Gross-Pikaevskii equation results is quite good. For these experimental runs, the length of the first pulse was adjusted such that half the population was in the state with zero momentum and half in the state with finite momentum. We emphasize that the resulting pulse 
length of $0.207 \mathrm{~ms}$ does not correspond to a $50 \%$ population transfer in the absence of interactions and vanishing momentum spread along the $z$-direction of the initial state. The second pulse was taken to be $0.200 \mathrm{~ms}$. To calibrate the coupling strength, we performed calculations for different $\Omega_{0, L}$ and picked the value that yields, using a $0.207 \mathrm{~ms}$ pulse, a 50/50 population distribution after the first pulse.

While the agreement between the symbols and solid lines in Fig. 10 is not perfect, the theoretical and experimental data share several key characteristics: (i) The number of fringes increases with increasing hold time. (ii) The density pattern is not characterized by a single fringe spacing; rather, the fringe spacings seem to vary across the expanded cloud. (iii) The density displays a small amplitude for $z$-values around $-75 \mu \mathrm{m}$ and $150 \mu \mathrm{m}$; these peaks correspond to momentum space components centered around $-2 \hbar k_{L}$ and $4 \hbar k_{L}$, respectively. (iv) The density distributions centered around $z \approx 0$ (corresponding to the component with momentum along the $z$-direction of $\approx 0$ ) and centered around $z \approx 75 \mu \mathrm{m}$ (corresponding to the component with momentum of $\approx 2 \hbar k_{L}$ ) have fairly distinct shapes, i.e., they are not mirror images of each other. All these observations are consistent with the discussion presented in the previous section. If the interaction effects played less of a role, the interference pattern would be "cleaner", i.e., more regular.

As already alluded to earlier, Ref. 25] measured the linear and quadratic phases using a Ramsey-type Bragg pulse sequence. Their analysis assumed equally spaced fringes. While the fringe pattern in Fig. 2 of Ref. 25] is more "regular" than the fringe pattern displayed in Fig. 10, the density peaks in Fig. 2(f) of Ref. 25] are, just as in our case, not fully symmetric with respect to the midpoint. We speculate that this might be due to the structural dynamics that is driven by mean-field effects ("factor of 2") discussed in our work for the lattice coupling case.

While the overall agreement between the experimental and theoretical data in Fig. 10 is satisfactory, the experimental data hint at the presence of beyond mean-field physics. In particular, we consistently observe a significant fraction of atoms "between" the two clouds, i.e., with a momentum of around $\hbar k_{L}$. It is presently unclear if this is due to quantum correlations that are not captured by the mean-field Gross-Pitaevskii equation or if, possibly, the thermal cloud plays a non-negligible role. A detailed investigation of these questions is beyond the scope of this work.

\section{SUMMARY AND OUTLOOK}

This paper investigated two realizations of a two-state model; in both realizations, the two states are represented by a spatially- and time-dependent mean-field wave function or orbital. The description goes beyond

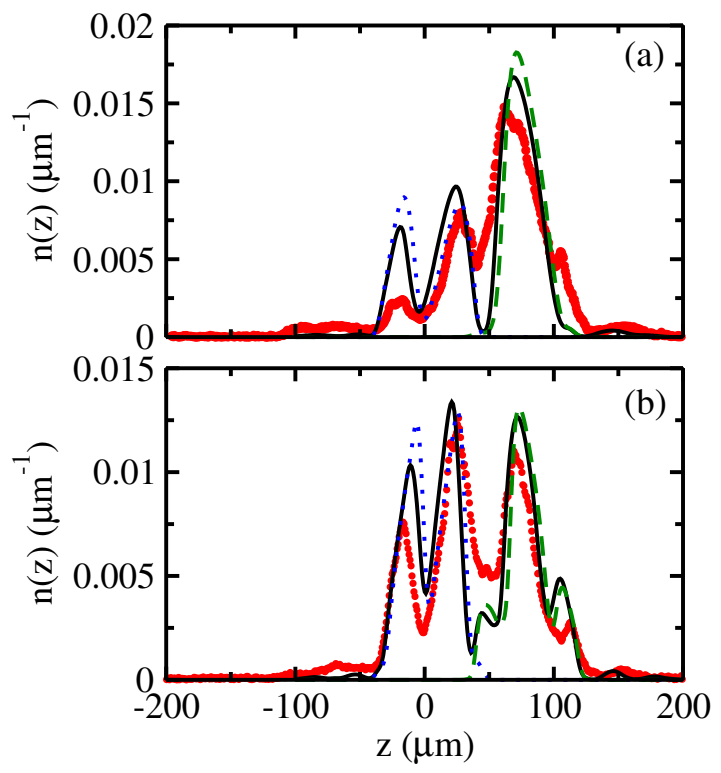

FIG. 10: Theory-experiment comparison for lattice Ramseytype pulse sequence for a ${ }^{87} \mathrm{Rb}$ BEC for two different hold times $t_{\text {hold }}$ and particle numbers $N$ : (a) $t_{\text {hold }}=0.5 \mathrm{~ms}$ $\left(N=3.8 \times 10^{5}\right)$ and (b) $t_{\text {hold }}=1 \mathrm{~ms}\left(N=4.3 \times 10^{5}\right)$. The red symbols show the experimentally measured integrated density $n(z), n(z)=\int|\Phi(\mathbf{r}, t)|^{2} d x d y$, as a function of $z$ for $t_{\text {start }}=0.5 \mathrm{~ms}, t_{\mathrm{ToF}}=12 \mathrm{~ms}, \delta_{L}=0$, and $E_{L} / h=1080 \mathrm{~Hz}$; the results shown are from a single experimental run. The solid black lines show results obtained by solving the Gross-Pitaevskii equation for the full lattice Hamiltonian for $\Omega_{0, L} / h=1372 \mathrm{~Hz}$. For comparison, the blue dotted and green dashed lines show results obtained from using the two-state model (see text). Both sets of theory data are convolved using a Gaussian with the experimentally measured resolution width of $2 \mu \mathrm{m}$. The pulse sequence is $\tau_{1}=0.207 \mathrm{~ms}$ (first $\pi / 2$-pulse), hold for time $t_{\text {hold }}$ (see above), and $\tau_{2}=0.200 \mathrm{~ms}$ (second $\pi / 2$-pulse). The experimentally determined trap frequencies are $\omega_{x}=2 \pi \times 119 \mathrm{~Hz}$, $\omega_{y}=2 \pi \times 163 \mathrm{~Hz}$, and $\omega_{z}=2 \pi \times 25.7 \mathrm{~Hz}$; the theory calculations set $\omega_{\rho}$ equal to the mean of $\omega_{x}$ and $\omega_{y}$.

a class of simpler mean-field models, where the dynamics of each mode is described by one complex number that encodes the population and phase of the mode, thereby assuming that the spatial dynamics of the modes play a negligible role 32 34. Our work demonstrates that time-dependent deformations of the spatial profile of the mean-field wave functions play an important role when the two-state model is realized by loading a singlecomponent ${ }^{87} \mathrm{Rb} \mathrm{BEC}$ into a moving one-dimensional optical lattice that introduces a coupling between two distinct momentum states of the atom. When the two-state model is, instead, realized by coupling two different hyperfine states of ${ }^{87} \mathrm{Rb}$ BEC atoms through a two-photon Raman process, time-dependent deformations of the spatial profile of the mean-field wave functions are notably less pronounced.

The difference in the dynamics for the two physical realizations (lattice and Raman coupling, respectively) of 
the two-mode model was traced back to the contribution of different scattering diagrams; in particular, there exist two scattering diagrams (these are depicted in the second row in Fig. 1) that contribute in the lattice coupling case but not in the Raman coupling case (due to the "factor of 2"). Said differently, the mean-field interactions for the lattice and Raman coupling cases differ: The effective two-state model for the lattice coupling case contains two repulsive "off-site" interaction terms that are absent in the Raman coupling case. As a consequence, the lattice coupled system is characterized by an enhanced tendency for phase separation, which "competes" with the lattice coupling term that has a tendency to keep the components together. This competition gives rise to the internal mean-field dynamics in the lattice coupled system that is different from the mean-field dynamics displayed by the two-state Hamiltonian for the Raman coupling case.

While the discussion throughout this paper focused on ${ }^{87} \mathrm{Rb} \mathrm{BECs}$, the lattice coupling results, which rely on the occupation of a single hyperfine state, apply to any BEC with positive two-body $s$-wave scattering length. The Raman coupling results were obtained assuming that the four coupling strengths $g_{a a}, g_{b b}, g_{a b}$, and $g_{b a}$ are approximately equal or equal to each other; this assumption holds for the $F=1$ states of ${ }^{87} \mathrm{Rb}$ but not necessarily for other elements.

The present work has a number of practical and conceptual implications:

- The Rabi oscillation data (see Figs. 2, 3, 7, and 8) show, especially for weak coupling strengths, pronounced non-sinusoidal behavior. This indicates that the analysis of experimental Rabi oscillation data, taken to calibrate the coupling strength, has to proceed with care. A simple fit to a sinusoidal function (or damped sinusoidal function) may yield an imprecise coupling strength due to interaction effects. Such data can be used for calibration purposes if compared with mean-field simulations that account for the interaction effects. Alternatively, experiments can operate in the dilute regime where interaction effects are negligible. Related discussions of lattice potential calibrations can be found in Refs. 28, 35 37.

- In the "weak" lattice coupling case - this is the regime where, as discussed in Sec. IV, the effective two-state model Hamiltonian provides a reliable description of the system dynamics - the internal dynamics leads to a deformation of the density profiles of the components. We argued that these density deformations can be interpreted as corresponding to an effective position-dependent detuning. For example, starting with all population in one of the two states, a $\pi / 2$-pulse (defined for a single atom in free space), realized using a comparatively weak coupling strength, yields a state with a population distribution that differs from a 50/50 mixture. This fact, together with the buildup of spatial deformations during the hold time, has implications for momentum space engineering protocols, which aim to implement beam-splitters and other operations that are commonly realized in quantum optics [17, 26].

- Integrating out the spatial degrees of freedom, the dynamics of the two-state Hamiltonian considered in this work reduces to coupled mean-field equations that are characterized by two complex numbers, representing the populations and phases of the two modes [32 34, 38. In the lattice case, this reduced dimensionality model has been shown to support intriguing swallow-tail lattice structures [32, 39 42, which support, e.g., mean-field induced non-exponential tunneling [32, 34]. The internal spatial dynamics highlighted in the present work suggests that the validity regime of these reduced dimensionality models needs to be assessed carefully.

\section{ACKNOWLEDGEMENT}

We thank Vandna Gokhroo for her contributions during the initial stage of this project. Support by the National Science Foundation through grant numbers PHY1806259 (QG and DB), PHY-1607495 (TMB, SM, and $\mathrm{PE}$ ), and PHY-1912540 (TMB, SM, and PE) are gratefully acknowledged. This work used the OU Supercomputing Center for Education and Research (OSCER) at the University of Oklahoma (OU).
[1] D. J. Griffiths, Introduction to Quantum Mechanics (Pearson Prentice Hall, 2005).

[2] L. Allen and J. H. Eberly, Two-level Atoms and Optical Resonance (Dover, 1987).

[3] I. I. Rabi, S. Millman, P. Kusch, and J. R. Zacharias, The molecular beam resonance method for measuring nuclear magnetic moments, Phys. Rev. 55, 526 (1939).

[4] T. A. Johnson, E. Urban, T. Henage, L. Isenhower, D. D. Yavuz, T. G. Walker, and M. Saffman, Rabi Oscillations between Ground and Rydberg States with Dipole-
Dipole Atomic Interactions, Phys. Rev. Lett. 100, 113003 (2008).

[5] H. De Raedt, B. Barbara, S. Miyashita, K. Michielsen, S. Bertaina, and S. Gambarelli, Quantum simulations and experiments on Rabi oscillations of spin qubits: Intrinsic vs extrinsic damping, Phys. Rev. B 85, 014408 (2012).

[6] C.-H. Li, C. Qu, R. J. Niffenegger, S.-J. Wang, M. He, D. B. Blasing, A. J. Olson, C. H. Greene, Y. Lyanda-Geller, Q. Zhou, C. Zhang, Y. P. Chen, Spin current generation and relaxation in a quenched spin-orbit-coupled Bose- 
Einstein condensate, Nat. Comm. 10, 375 (2019).

[7] Y.-J. Lin, K. Jiménez-García and I. B. Spielman, Spinorbit-coupled Bose-Einstein condensates, Nature 471, 83 (2011).

[8] V. Galitski and I. B. Spielman, Spin-orbit coupling in quantum gases, Nature 494, 49 (2013).

[9] H. Zhai, Degenerate quantum gases with spin-orbit coupling: a review, Rep. Prog. Phys. 78, 026001 (2015).

[10] M. B. Dahan, E. Peik, J. Reichel, Y. Castin, and C. Salomon, Bloch Oscillations of Atoms in an Optical Potential, Phys. Rev. Lett. 76, 4508 (1996).

[11] M. Kozuma, L. Deng, E. W. Hagley, J. Wen, R. Lutwak, K. Helmerson, S. L. Rolston, and W. D. Phillips, Coherent Splitting of Bose-Einstein Condensed Atoms with Optically Induced Bragg Diffraction, Phys. Rev. Lett. 82, 871 (1999).

[12] O. Morsch, J. H. Müller, M. Cristiani, D. Ciampini, and E. Arimondo, Bloch Oscillations and Mean-Field Effects of Bose-Einstein Condensates in 1D Optical Lattices, Phys. Rev. Lett. 87, 140402 (2001).

[13] N. Ramsey, Molecular Beams (Clarendon, 1985).

[14] Y. Castin and R. Dum, Bose-Einstein Condensates in Time Dependent Traps, Phys. Rev. Lett. 77, 5315 (1996).

[15] P. B. Blakie and R. J. Ballagh, Mean-field treatment of Bragg scattering from a Bose-Einstein condensate, J. Phys. B 33, 3961 (2000).

[16] A. O. Jamison, J. N. Kutz, and S. Gupta, Atomic interactions in precision interferometry using Bose-Einstein condensates, Phys. Rev. A 84, 043643 (2011).

[17] M. Edwards, B. Benton, J. Heward, and C. W. Clark, Momentum-space engineering of gaseous Bose-Einstein condensates, Phys. Rev. A 82, 063613 (2010).

[18] B. Gadway, Atom-optics approach to studying transport phenomena, Phys. Rev. A 92, 043606 (2015).

[19] C. Qu, C. Hamner, M. Gong, C. Zhang, and P. Engels, Observation of Zitterbewegung in a spin-orbit-coupled Bose-Einstein condensate, Phys. Rev. A 88, 021604(R) (2013).

[20] C. Hamner, Y. Zhang, M. A. Khamehchi, M. J. Davis, and P. Engels, Spin-Orbit-Coupled Bose-Einstein Condensates in a One-Dimensional Optical Lattice, Phys. Rev. Lett. 114, 070401 (2015).

[21] The values of the scattering lengths are taken from $\mathrm{M}$. A. Khamehchi, Y. Zhang, C. Hamner, T. Busch, and P. Engels, Measurement of collective excitations in a spinorbit-coupled Bose-Einstein condensate, Phys. Rev. A 90, 063624 (2014).

[22] H. Tal-Ezer, and R. Kosloff, An accurate and efficient scheme for propagating the time dependent Schrödinger equation, J. Chem. Phys. 81, 3967 (1984).

[23] C. Leforestier, R. H. Bisseling, C. Cerjan, M. D. Feit, R. Friesner, A. Guldberg, A. Hammerich, G. Jolicard, W. Karrlein, H. D. Meyer, N. Lipkin, O. Roncero, and R. Kosloff, A Comparison of Different Propagation Schemes for the Time Dependent Schrödinger Equation, J. Comput. Phys. 94, 59 (1991).

[24] F. Dalfovo, S. Giorgini, L. P. Pitaevskii, and S. Stringari, Theory of Bose-Einstein condensation in trapped gases, Rev. Mod. Phys. 71, 463 (1999).

[25] J. E. Simsarian, J. Denschlag, M. Edwards, C. W. Clark,
L. Deng, E. W. Hagley, K. Helmerson, S. L. Rolston, and W. D. Phillips, Imaging the Phase of an Evolving BoseEinstein Condensate Wave Function, Phys. Rev. Lett. 85, 2040 (2000).

[26] B. Benton, M. Krygier, J. Heward, M. Edwards, and C. W. Clark, Prototyping method for Bragg-type atom interferometers, Phys. Rev. A 84, 043648 (2011).

[27] K. Bongs, S. Burger, S. Dettmer, D. Hellweg, J. Arlt, W. Ertmer, and K. Sengstock, Waveguide for Bose-Einstein condensates, Phys. Rev. A 63, 031602(R) (2001).

[28] O. Morsch and M. Oberthaler, Dynamics of BoseEinstein condensates in optical lattices, Rev. Mod. Phys. 78, 179 (2006).

[29] E. Timmermans, Phase Separation of Bose-Einstein Condensates, Phys. Rev. Lett. 81, 5718 (1998).

[30] C. V. Parker, L.-C. Ha, and C. Chin, Direct observation of effective ferromagnetic domains of cold atoms in a shaken optical lattice, Nat. Phys. 9, 769 (2013).

[31] F. A. An, E. J. Meier, J. Ang'ong'a, and B. Gadway, Correlated Dynamics in a Synthetic Lattice of Momentum States, Phys. Rev. Lett. 120, 040407 (2018).

[32] B. Wu and Q. Niu, Nonlinear Landau-Zener tunneling, Phys. Rev. A 61, 023402 (2000).

[33] O. Zobay and B. M. Garraway, Time-dependent tunneling of Bose-Einstein condensates, Phys. Rev. A 61, 033603 (2000).

[34] J. Liu, L. Fu, B.-Y. Ou, S.-G. Chen, D.-I. Choi, B. Wu, and Q. Niu, Theory of nonlinear Landau-Zener tunneling, Phys. Rev. A 66, 023404 (2002).

[35] M. Cristiani, O. Morsch, J. H. Müller, D. Ciampini, and E. Arimondo, Experimental properties of Bose-Einstein condensates in one-dimensional optical lattices: Bloch oscillations, Landau-Zener tunneling, and mean-field effects, Phys. Rev. A 65, 063612 (2002).

[36] C. Cabrera-Gutiérrez, E. Michon, V. Brunaud, T. Kawalec, A. Fortun, M. Arnal, J. Billy, and D. GuéryOdelin, Robust calibration of an optical-lattice depth based on a phase shift, Phys. Rev. A 97, 043617 (2018).

[37] B. T. Beswick, I. G. Hughes, and S. A. Gardiner, Lattice-depth measurement using continuous grating atom diffraction, Phys. Rev. A 100, 063629 (2019).

[38] M. Jona-Lasinio, O. Morsch, M. Cristiani, N. Malossi, J. H. Müller, E. Courtade, M. Anderlini, and E. Arimondo, Asymmetric Landau-Zener Tunneling in a Periodic Potential, Phys. Rev. Lett. 91, 230406 (2003).

[39] K. Berg-Sørensen and K. Mølmer, Bose-Einstein condensates in spatially periodic potentials, Phys. Rev. A 58, 1480 (1998).

[40] B. Wu, R. B. Diener, and Q. Niu, Bloch waves and bloch bands of Bose-Einstein condensates in optical lattices, Phys. Rev. A 65, 025601 (2002).

[41] D. Diakonov, L. M. Jensen, C. J. Pethick, and H. Smith, Loop structure of the lowest Bloch band for a BoseEinstein condensate, Phys. Rev. A 66, 013604 (2002).

[42] M. Machholm, C. J. Pethick, and H. Smith, Band structure, elementary excitations, and stability of a BoseEinstein condensate in a periodic potential, Phys. Rev. A 67, 053613 (2003). 